\title{
Forschungsstand zu Migrantenselbstorganisationen
}

\subsection{Migrantenselbstorganisationen in Zahlen}

Die Anzahl der in Deutschland existierenden MSO wurde lange Zeit nur sehr grob geschätzt und auf 10.000 bis 20.000 beziffert (Pries 2013a: 2; IntB 2011: 6). Viel zitiert wurde die von Uwe Hunger ermittelte Zahl von 16.000 Vereinen (Hunger 2005: 226 ff.), die auf Basis seiner Auswertung des Ausländervereinsregisters ${ }^{1}$ für das Jahr 2001 zustande kam; circa 11.000 konnten damals als von Türkeistämmigen dominiert betrachtet werden (vgl. Tab. 2.1). Anhand der Analyse von Hunger konnten insgesamt 148 Nationalitäten ausgemacht und eine sehr hohe Anzahl ausländischer Vereine in Nordrhein-Westfalen (38 \%) festgestellt werden; in ostdeutschen Bundesländern waren Ausländervereine aufgrund der geringen Ansiedlungsdichte von Menschen mit Zuwanderungsgeschichte hingegen kaum zu registrieren (Hunger 2005: 226 ff.).

Hinsichtlich der in den Satzungen festgelegten, formal am häufigsten genannten Vereinszwecke ergab sich für das Jahr 2001 folgendes Ranking: „1. Kultur, 2. Begegnung, 3. Religion, 4. Sport, 5. Beratung, 6. Betreuung, 7. Politik, 8. Bildung, 9. Humanitäres, 10. Freizeit" (Pries 2013a: 3; Tab. 2.2). Eine genauere Betrachtung der jeweiligen Vereinsausrichtung ergab, dass nichtchristliche Bevölkerungsgruppen wie etwa Türken, Koreaner und Inder primär religiöse Vereine

\footnotetext{
${ }^{1}$ Das vom Bundesverwaltungsamt geführte sogenannte Ausländervereinsregister registrierte bis zum Jahr 2001 alle in Deutschland von Personen mit einer nichtdeutschen Staatsangehörigkeit gegründeten Vereine. Seit 2001 werden in das Register nur noch Vereine von Drittstaatsangehörigen aufgenommen (Friedrichs et al. 2020: 12; Pries 2010a). Das Ausländervereinsregister existiert aufgrund einer gesonderten Meldepflicht für Drittstaaten-Ausländervereine und dient der ,präventiven Gefahrenabwehr“; URL: https://www.bva.bund.de/SharedDocs/ Aufgaben/DE/A/auslaendervereinsregister.html (letzter Abruf am 03.04.2021).
} 
gegründet hatten, während bei den anderen der Typus des Kultur- und Begegnungsvereins dominierte. In Bezug auf die spanische Bevölkerungsgruppe war im Vergleich zu den anderen Nationen zudem die hohe Anzahl von Elternvereinen auffällig (ebd.: 236).

Tab. 2.1 Häufigste Nationalitäten von Ausländervereinen 2001 (mit n>100)

\begin{tabular}{lcc} 
& Häufigkeit & Prozent \\
\hline Türkei & 1.130 & 19,5 \\
\hline Griechenland & 468 & 8,1 \\
\hline Italien & 411 & 7,1 \\
\hline Spanien & 298 & 5,2 \\
\hline Iran & 204 & 3,5 \\
\hline ehem. Jugoslawien & 195 & 3,4 \\
\hline Kroatien & 146 & 2,5 \\
\hline Bosnien-Herzegowina & 124 & 2,1 \\
\hline Portugal & 140 & 2,4 \\
\hline Marokko & 123 & 2,1 \\
\hline Afghanistan & 101 & 1,7 \\
\hline
\end{tabular}

Quelle: Hunger 2005: 227; Datenbasis: Bundesverwaltungsamt, Zufallsstichprobe.

Seit der Auswertung von Hunger haben sich bundesweit - auf Basis anderer Datengrundlagen - zahlenmäßig erfassbare Veränderungen ergeben. Der im Jahr 2017 vorgelegte zweite ZiviZ-Survey² (ZiviZ: „Zivilgesellschaft in Zahlen") kommt anhand einer bundesweiten Vereinsliste von über 600.000 Vereinen, auf Basis einer Namensanalyse, auf eine Anzahl von 17.414 MSO, unter denen Organisationen verstanden werden, deren „Mitglieder überwiegend Menschen mit Migrationshintergrund sind" (Priemer 2017: 1) ${ }^{3}$. Diese Zahl entspricht einem Anteil von circa 3 Prozent aller in Deutschland eingetragenen Vereine. Auf Basis der Sondierung ist erkennbar, dass in den letzten Jahren zahlreiche neue MSO

${ }^{2}$ Der ZiviZ-Survey ist eine Befragung, die sich an alle eingetragenen Vereine, Stiftungen, Genossenschaften und Gesellschaften mit beschränkter Haftung (GmbHs) in Deutschland richtet.

${ }^{3}$ Im Rahmen des ZiviZ-Surveys 2017 wurden über das bundesweite Vereinsregister per Namensanalyse MSO identifiziert (Datenbasis: Vereinsregister 2016; gemeinsames Registerportal der Länder, Suchabfragen unter: https://www.handelsregister.de) (Priemer u. a. 2017: $41,50)$. 
Tab. 2.2 Primärer Vereinszweck von Ausländervereinen 2001

\begin{tabular}{lcc}
\hline & Häufigkeit & Prozent \\
\hline Kultur & 1.376 & 25,5 \\
\hline Begegnung & 919 & 17,0 \\
\hline Religion & 531 & 9,8 \\
\hline Sport & 504 & 9,3 \\
\hline Beratung & 473 & 8,8 \\
\hline Betreuung & 408 & 7,6 \\
\hline Politik & 289 & 5,4 \\
\hline Bildung & 218 & 4,0 \\
\hline Humanitär & 188 & 3,5 \\
\hline Freizeit & 169 & 3,1 \\
\hline Integration & 134 & 2,5 \\
\hline Jugendarbeit & 105 & 1,9 \\
\hline Frauenarbeit & 39 & 0,7 \\
\hline Gesundheit & 31 & 0,6 \\
\hline Seniorenarbeit & 8 & 0,1 \\
\hline Gesamt & 5.392 & 100,0 \\
\hline
\end{tabular}

Quelle: Hunger 2005: 231; Datenbasis: Bundesverwaltungsamt, Zufallsstichprobe.

Ein Verein wurde dann als MSO kategorisiert, wenn im Namen ein Raumbezug (z. B. „türkisch“ in ,Türkischer Kulturverein Dortmund e. V.“) oder ein sich nicht auf die Mehrheitsgesellschaft beziehender Religionsbezug (z. B. ,islamisch“) erkennbar war. Darüber hinaus wurden fremdsprachige Namen (z. B. Merkez Osmanlı Camii e. V.) und weitere Begrifflichkeiten, die auf einen Migrationsbezug verweisen könnten (wie z. B. „Migration“ oder „,internationale Solidarität“), bei der Filterung berücksichtigt (Priemer/Schmidt 2018: 5). An dieser Stelle ist anzumerken, dass die Namensanalyse ein forschungspragmatisches, aber nicht hinreichend präzises Analysekriterium darstellt. Denn Voraussetzung für die Zählung eines Vereins als eine MSO ist bei dieser Vorgehensweise der im Vereinsnamen deutlich werdende Migrations-/Länderbezug, der Vereinigungen ohne eine solche Explikation in der Listung unberücksichtigt lässt. Zum anderen muss ein solcher Namensbezug nicht zwangsläufig bedeuten, dass es sich um eine entsprechend der gängigen Definitionskriterien zu klassifizierende MSO handelt; stattdessen kann es sich um binationale oder entwicklungspolitische Zusammenschlüsse handeln, die allerdings nicht von Menschen mit einem Migrationshintergrund getragen werden (vgl. Friedrichs et al. 2020: 12). Nicht zuletzt zeigte auch die Recherche von Kontaktdaten von MSO durch die Autorin der vorliegenden Arbeit (III. 5.3), 
gegründet wurden: 16 Prozent sind erst in den letzten fünf Jahren in die Vereinsregister eingetragen worden, was auf die wachsende Anzahl von in Deutschland lebenden Menschen mit Migrationshintergrund zurückzuführen ist (Priemer et al. 2017: 41). Im Allgemeinen ist für die Gründungs- und Entwicklungsprozesse der MSO eine hohe Dynamik und Fluktuation auszumachen (Priemer/Schmidt 2018). Seit den 2000er Jahren sind zahlreiche Neugründungen zu konstatieren, die jedoch von Wiederauflösungen flankiert werden (Friedrichs et al. 2020: 19; III. 5.3).

Mit der Studie Vielfältig engagiert - breit vernetzt - partiell eingebunden? Migrantenorganisationen als gestaltende Kraft in der Gesellschaft des SVRForschungsbereichs (Friedrichs et al. 2020) liegen für Deutschland die aktuellsten Daten zu MSO vor. Auf Basis einer Vollerhebung bzw. Erstellung einer Datenbank für die in den Bundesländern Nordrhein-Westfalen, Bayern, Sachsen und Berlin (als Modellregionen) insgesamt gezählten 6.851 MSO erfolgte eine ,vorsichtige" (ebd.: 11) statistische Schätzung der Gesamtanzahl von MSO in Deutschland, der zufolge derzeit zwischen 12.400 und 14.300 aktive, als Verein eingetragene MSO existieren (ebd.: 13, 15) ${ }^{4}$. Für die Schätzung der Studie des SVR-Forschungsbereichs ist herauszustellen, dass Organisationen, die nicht den Definitionskriterien der $\mathrm{MSO}^{5}$ entsprochen haben, nicht in die Datenbank aufgenommen wurden und sich die statistischen Schätzungen ausschließlich auf aktive MSO beziehen (ebd.: 15). Die im Vergleich zu anderen Schätzungen geringer ausfallende Anzahl (für das Jahr 2001: 16.000 MSO, Hunger 2005; für 2017: 17.414, Priemer 2017) ist mit der Verwendung von verschiedenen Datenquellen und unterschiedlichen methodischen Zugriffen zu erklären ${ }^{6}$ (vgl. dazu auch Friedrichs et al. 2020: 15).

dass sich in verfügbaren Listen und offiziellen Registern zahlreiche Vereine befinden, die sich zwischenzeitlich wieder aufgelöst haben, bei denen es sich also um „Karteileichen“ handelt.

${ }^{4}$ Insgesamt wurden für die Bundesländer acht statistische Modelle berechnet und folgende Einflussfaktoren berücksichtigt: Anzahl der Personen mit Migrationshintergrund und der weiblichen Einwohnerinnen; Anzahl der Einwohner $/ \mathrm{km}^{2}$; Anzahl der Einbürgerungen; Bruttoinlandsprodukt (BIP) pro Kopf; Fläche des Bundeslandes; Faktor Ost-/Westdeutschland (Friedrichs et al. 2020: 13).

${ }^{5}$ Im Rahmen seiner Erhebung versteht der SVR-Forschungsbereich MSO als ,,(1) gemeinnützige Zusammenschlüsse, (2) die mindestens zur Hälfte von Menschen mit Zuwanderungsgeschichte getragen werden oder von entsprechenden Personen gegründet wurden und (3) bei denen für ihr Selbstverständnis, ihre Ziele und Aktivitäten eine Migrationserfahrung im weitesten Sinne zentral ist" (Friedrichs et al. 2020: 9).

${ }^{6}$ Hunger 2005: Zählung der MSO auf Basis der Einträge im Ausländervereinsregister; Priemer et al. 2017: Zählung der MSO auf Basis einer Analyse von Vereinsnamen im deutschen Vereinsregister; Friedrichs et al. 2020: statistische Schätzung, d. h. Berechnung von multivariaten linearen Regressionsmodellen, auf Basis einer Vollerhebung in vier Bundesländern. 
Die Auswertung nach Bundesländern zeigt sowohl für 2001 als auch für die Jahre 2016 und 2019, dass die meisten der MSO in den vier bevölkerungsstarken Bundesländern Nordrhein-Westfalen, Baden-Württemberg, Bayern und Hessen mit hohen Bevölkerungsanteilen von Personen mit Migrationshintergrund ansässig sind; es folgen Niedersachsen, Berlin, Rheinland-Pfalz und Hamburg, die in den beiden benannten Jahren hinsichtlich zahlenmäßiger Verhältnisse jeweils unterschiedliche Ränge einnehmen (Tab. 2.3). Schleswig-Holstein, Saarland und Bremen weisen unter den westdeutschen Bundesländern die geringste Anzahl von MSO auf. In den fünf ostdeutschen Bundesländern sind insgesamt die wenigsten MSO beheimatet, wobei Sachsen jedoch vor Saarland und Bremen rangiert und Mecklenburg-Vorpommern 2016 das Schlusslicht bildet.

Den Analysen der SVR-Studie zufolge ist die Quantität von MSO in einer Region proportional abhängig von der Anzahl der dort lebenden Personen mit einer Zuwanderungsgeschichte. Für NRW lässt sich mit einer zunehmenden Bevölkerungsdichte eine höhere Zahl von MSO feststellen und für Bayern ist zu konstatieren, dass die Zahl der MSO sinkt, je höher die Einbürgerungsquote von Zugewanderten ausfällt (Friedrichs et al. 2020: 14). Für Ostdeutschland ist die Anzahl der MSO im Verhältnis zur Bevölkerung mit Migrationshintergrund als hoch und „deutlich höher als in den westdeutschen Flächenländern“ (ebd.: 16) einzustufen.

Die Autorin dieser Arbeit hat auf Basis der Analyse verfügbarer Datensätze - anhand der MSO-Listung des ZiviZ-Surveys für NRW (Vereinsregister, Stand: 2016) und mithilfe des im Jahr 2017 aktualisierten Datenpools des Forschungsprojekts „Religiöse Vielfalt in Nordrhein-Westfalen“ (Hero et al. 2008) - explorativ eine grobe Zuordnung der in NRW existierenden MSO zu religiösen Traditionen vorgenommen. ${ }^{7}$

\footnotetext{
${ }^{7}$ Siehe Fußnote 3. Im Datenpool des Forschungsprojekts , Religiöse Vielfalt in NordrheinWestfalen“ (2017) sind religiöse Ausrichtungen kategorisiert, sodass Migrationsreligionen herausgefiltert werden konnten.
} 
Tab. 2.3 Verteilung von migrantischen bzw. ausländischen Vereinen auf die Bundesländer 2019, 2016 und 2001

\begin{tabular}{|c|c|c|c|c|c|c|c|c|}
\hline Bundesland & $\begin{array}{c}\text { Anzahl } \\
2019\end{array}$ & $\begin{array}{c}\text { Anzahl } \\
2016\end{array}$ & in $\%$ & Rang & $\begin{array}{c}\text { Anzahl } \\
2001\end{array}$ & in $\%$ & Rang & $\begin{array}{c}\text { Pers. mit } \\
M^{\star *} 2018(\%)\end{array}$ \\
\hline Nordrhein-Westfalen & 4.122 & 5.078 & 29,2 & 1 & 2.152 & 37,5 & 1 & 30,4 \\
\hline Baden-Württemberg & $\begin{array}{l}750- \\
2.500^{*}\end{array}$ & 3.154 & 18,1 & 2 & 935 & 16,3 & 2 & 33,4 \\
\hline Bayern & 1.766 & 2.217 & 12,7 & 3 & 730 & 12,7 & 3 & 25,6 \\
\hline Hessen & $\begin{array}{l}750- \\
2.500^{*}\end{array}$ & 1.706 & 9,8 & 4 & 726 & 12,6 & 4 & 33,6 \\
\hline Niedersachsen & $\begin{array}{l}750- \\
2.500^{*}\end{array}$ & 1.501 & 8,6 & 5 & 254 & 4,4 & 7 & 22,1 \\
\hline Berlin & 732 & 1.118 & 6,4 & 6 & 332 & 5,8 & 5 & 31,6 \\
\hline Rheinland-Pfalz & $150-750^{*}$ & 791 & 4,5 & 7 & 120 & 2,1 & 8 & 26,1 \\
\hline Hamburg & $150-750^{*}$ & 451 & 2,6 & 8 & 323 & 5,6 & 6 & 33,3 \\
\hline Schleswig-Holstein & $150-750^{*}$ & 377 & 2,2 & 9 & 52 & 0,9 & 11 & 17,3 \\
\hline Sachsen & 231 & 272 & 1,6 & 10 & 2 & 0,0 & 13 & 8,2 \\
\hline Saarland & $0-150^{*}$ & 194 & 1,1 & 11 & 56 & 1,0 & 9 & 22,5 \\
\hline Bremen & $150-750^{*}$ & 179 & 1,0 & 12 & 54 & 0,9 & 10 & 35,1 \\
\hline Thüringen & $0-150^{*}$ & 110 & 0,6 & 13 & 6 & 0,1 & 12 & 7,3 \\
\hline Sachsen-Anhalt & $0-150^{*}$ & 108 & 0,6 & 14 & 0 & 0,0 & 15 & 7,8 \\
\hline Brandenburg & $0-150^{*}$ & 107 & 0,6 & 15 & 2 & 0,0 & 13 & 8,4 \\
\hline $\begin{array}{l}\text { Mecklenburg- } \\
\text { Vorpommern }\end{array}$ & $0-150^{*}$ & 51 & 0,3 & 16 & 1 & 0,0 & 14 & 7,9 \\
\hline Gesamt & $\begin{array}{l}12.400- \\
14.300^{*}\end{array}$ & 17.414 & 100,0 & & 5.745 & 100,0 & & \\
\hline
\end{tabular}

Quelle: Friedrichs et al. 2020: 13-15, Datenbasis: Vollerhebung in vier Modellregionen (Bundesländern) Nordrhein-Westfalen, Baden-Württemberg, Sachsen, Berlin 2019; Priemer et al. 2017: 41, 50, Datenbasis: Bundesweites Vereinsregister 2016; Hunger 2005: 225, Datenbasis: Ausländervereinsregister 2001; Destatis 2019b (Ergebnisse des Mikrozensus: Bevölkerung in Privathaushalten 2018 nach Migrationshintergrund; eigene Berechnung des Quotienten aus der absoluten Zahl der Menschen mit MH und der Gesamtbevölkerung des jeweiligen Bundeslandes).

* Schätzungen auf Basis der Berechnung multivariater Regressionsmodelle $\mid * * \mathrm{MH}=$ Migrationshintergrund. 
Anhand der Datenintegration lassen sich insgesamt 1.384 (32\%) der MSO als religiöse kategorisieren. ${ }^{8}$ Von diesen sind 53 Prozent islamisch, von denen wiederum 19 Prozent DİTİB und 7 Prozent Millî Görüş angehören. Zum Christentum wird sich in 38 Prozent der religiösen Vereinigungen bekannt; bei 14 Prozent handelt es sich um christlich-orthodoxe und bei 7 Prozent - und damit unter den orthodoxen mehrheitlich - wiederum um eine griechisch-orthodoxe Ausrichtung. 11 Prozent der christlichen Vereinigungen sind evangelikalen und 6 Prozent pfingstlich-charismatischen Orientierungen zuzuordnen. Dem Alevitentum ${ }^{9}$ lassen sich 4 Prozent der Organisationen, dem Judentum und dem Hinduismus jeweils 2 Prozent und dem Buddhismus lediglich 1 Prozent der religiösen Vereinigungen zuordnen. Die Zahlen sind nicht unumstößlich, sondern vielmehr als eine quantitative Annäherung an die Erfassung der religiösen Pluralität in der Landschaft der MSO zu betrachten, zumal sich die Datenlage als lückenhaft erweist (z. B. lassen sich lediglich 3 ezidische Vereinigungen identifizieren).

Insgesamt ist die quantitative Datenlage zu MSO - ihre Anzahl, ihre Aktivitäten, ihre Zusammensetzung, ihre Gesamtsituation betreffend - als dünn zu betrachten. Aktuelle Daten zu MSO wurden mit dem ZiviZ-Survey 2017 (Priemer et al. 2017) und der im Dezember 2020 vom SVR-Forschungsbereich veröffentlichten Studie Vielfältig engagiert - breit vernetzt - partiell eingebunden? (Friedrichs et al. 2020) vorgelegt.

In den folgenden Abschnitten werden einige Ergebnisse dieser beiden MSOStudien berichtet. Allerdings ist anzumerken, dass die Vergleichbarkeit der Befunde aufgrund der Nutzung von unterschiedlichen Datensätzen, aufgrund verschiedener methodischer Zugriffe und Definitionskriterien für MSO wie auch

\footnotetext{
${ }^{8}$ Anmerkung zu dieser separaten Auswertung durch die Autorin dieser Arbeit: Im Rahmen der Analyse für NRW reduzierte sich die Gesamtanzahl von MSO auf (ca.) 4.383, denn bei einem genaueren Blick in die Gesamtliste zeigte sich, dass die für den ZiviZ-Survey per Namensanalyse für NRW herausgefilterten MSO auch solche umfasste, deren Bezeichnung nicht eindeutig auf den Charakter einer MSO schließen lässt (s. dazu auch Fußnote 3). Diesbezüglich exemplarisch anzuführen wären etwa folgende Vereine: ABC für indische Kinder e. V.; Afghanische Kinderhilfe Deutschland e. V.; Afrika Hilfe e. V.; Caritas-Flüchtlingshilfe Essen e. V.; Förderverein Tschernobyl-Kinder in Petuchowka e. V.; Amerikanische Hütehunde Europa e. V. Eine genaue Prüfung, ob es sich um MSO handelt, war aus zeitlichen Gründen nicht möglich und hätte einer separaten Projektdurchführung entsprochen. Vereine mit solchen Bezeichnungen wurden aus dem Datensatz entfernt.

${ }^{9}$ Entsprechend der Organisationsbezeichnungen werden muslimische und alevitische MSO hier getrennt aufgeführt. Damit ist ausdrücklich keine Kennzeichnung einer Nichtzugehörigkeit der Aleviten zum Islam beabsichtigt. Ob sich Aleviten dem Islam zugehörig fühlen und sich als Muslime bezeichnen, ist ein inneralevitisch kontrovers diskutiertes, im Rahmen dieser Arbeit aber nicht weiter auszuführendes Thema.
} 
unterschiedlicher Frageformulierungen, Antwortvorgaben und Operationalisierungen in den jeweils verwendeten Erhebungsinstrumenten eingeschränkt ist.

Mit der Auswertung der Daten von 121 MSO (2 \% der Stichprobe) im Rahmen des ZiviZ-Surveys 2017 (Priemer et al. 2017; Priemer 2017; Priemer/Schmidt 2018) liegen zwar relativ aktuelle, aber keine repräsentativen Daten vor. ${ }^{10}$ Mit der Erhebung des SVR-Forschungsbereichs (Friedrichs et al. 2020) konnten hingegen wesentlich mehr MSO erreicht werden. Sie basiert auf der Kombination eines quantitativen und qualitativen Forschungsansatzes, indem in den vier Bundesländern Nordrhein-Westfalen, Bayern, Sachsen und Berlin mit einer standardisierten Online-Befragung insgesamt 764 MSO und in 17 leitfadengestützten Interviews zusätzlich Vertreter von ausgewählten MSO befragt wurden (ebd.: 6). In ähnlicher Weise wie die für die vorliegende Arbeit in Nordrhein-Westfalen durchgeführte Studie hat sich die Untersuchung des SVR zum Ziel gesetzt, im Rahmen einer Vollerhebung in den vier genannten Bundesländern die Aktivitätsfelder, Selbstverständnisse, Funktionswahrnehmungen, finanzielle Situation sowie Kooperationen der MSO zu erfassen. Die Studie wurde in den Jahren 2019/2020 parallel zu der dieser Arbeit zugrunde liegenden Erhebung in NRW durchgeführt.

Der Auswertung des ZiviZ-Surveys zufolge gibt es die meisten MSO in urbanen (Ballungs-)Gebieten mit vielen Einwohnern, mit einer hohen Bevölkerungsdichte sowie einem hohen Ausländeranteil (Priemer/Schmidt 2018: 2). Mit rund 72 Prozent sind MSO hauptsächlich und viel häufiger als nicht-migrantische Organisationen (33 \%) in Großstädten mit mehr als 100.000 Einwohnern ansässig (Priemer/Schmidt 2019). Laut der SVR-Studie handelt es sich bei dem dominanten Tätigkeitsbereich der MSO mit circa 61 Prozent hauptsächlich um die eigene Stadt oder den eigenen Stadtbezirk und weitere 14 Prozent sind in mehreren Kommunen bzw. Gemeinden aktiv. Weitere Aktivitätsbereiche betreffen ein einzelnes Bundesland ( $8 \%$ ), mehrere Bundesländer (4\%), ganz Deutschland (5\%) und den internationalen Raum (7 \%) (Friedrichs et al. 2020: 17 f.).

Die SVR-Studie präsentiert das Bild einer stark ausdifferenzierten Landschaft der MSO, in denen die untersuchten Vereinigungen vielen Aktivitätsfeldern gleichzeitig nachgehen. Der Erhebung zufolge stellt der Austausch zwischen Menschen mit und ohne Zuwanderungsgeschichte für das Jahr 2019 mit rund 45 Prozent die am häufigsten ausgeführte Tätigkeit dar (bei maximal fünf möglichen Antworten). Weitere Schwerpunkte liegen im sozialen Bereich, wie etwa in der

${ }^{10}$ Aufgrund der geringen Fallzahl der MSO sollten die Ergebnisse des ZiviZ-Surveys nicht überbewertet werden. Zudem ist anzunehmen, dass sich insbesondere innerhalb der adressierten MSO eingebundene Personen mit guten deutschen Sprachkenntnissen an der Umfrage beteiligt haben, sodass ein entsprechender Bias nicht auszuschließen ist (Priemer 2017: 1). 
Kinder- und Jugendarbeit (45\%), im Bildungsbereich (42\%), in künstlerischkulturellen Aktivitäten (39\%), in der Unterstützung von Geflüchteten (36\%) sowie in Beratungsangeboten $(33 \%)$ (ebd.: 23 f. $)^{11}$. Im Vergleich zu nichtmigrantischen Organisationen liegt ein Schwerpunkt der Tätigkeiten der MSO damit in der sozialen Arbeit (ebd.: 26). Im Vergleich der Aktivitäten der MSO in den west- und ostdeutschen Bundesländern zeigt sich, dass die MSO in letzteren einen Aktivitätsschwerpunkt auf unterschiedliche Formate des interkulturellen Austausches (57 \%) legen, was auf die erhöhte Fluchtzuwanderung in die ostdeutschen Bundesländer in den letzten Jahren zurückgeführt wird (ebd.: 25).

Über 61 Prozent der MSO, die Geflüchtete unterstützen (36\%), sind laut der Erhebung des SVR-Forschungsbereiches bereits seit ihrer Gründung in der Flüchtlingshilfe aktiv und 35 Prozent haben ihre Angebote für Geflüchtete im Laufe der Zeit ausgeweitet bzw. geöffnet. Zum Angebotsrepertoire in der Geflüchtetenhilfe gehören insbesondere Begleitangebote (41\%), etwa bei Behörden- oder Arztgängen. Zudem stellt die Unterstützung von Kontaktaufnahmen zu anderen zugewanderten oder geflüchteten Menschen (40\%) ein zentrales Anliegen der MSO dar. Weitere Angebote bestehen in der Unterstützung bei der Erstorientierung (35\%), beim Übersetzen bzw. Dolmetschen (29\%), bei der Beantragung von Leistungen (19\%), der Arbeitsmarktintegration (16\%) und der Wohnungssuche (11\%) (ebd.: 29 ff.).

Den Befunden der SVR-Studie entsprechend sind MSO auch nach den Ergebnissen des ZiviZ-Surveys häufig im Bereich der Kulturvermittlung aktiv: 84 Prozent der MSO organisieren auf lokaler Ebene interkulturellen Austausch (oft: $49 \%$, manchmal: $35 \%$ ) und jede zweite MSO ist im internationalen Austausch involviert (Priemer et al. 2017: 44; Priemer 2017: 2). Zudem engagieren sich 63 Prozent der MSO - und damit ähnlich viele wie der SVR-Studie zufolge - für geflüchtete Menschen. Weitere Tätigkeitsbereiche stellen Bildung und Erziehung (24\%), Kultur (23\%), Religion (20\%) sowie Sport (10\%) dar. Die drei letztgenannten Bereiche befanden sich auch bei der Analyse von Uwe Hunger unter den am häufigsten aufgeführten Vereinszwecken (Tab. 2.2). Dem ZiviZ-Survey zufolge sind jüngere MSO häufiger in den Bereichen Bildung, soziale Dienste und internationale Solidarität aktiv und sie betrachten sich selbst

\footnotetext{
${ }^{11}$ Weitere Tätigkeiten umfassen Angebote für Frauen (26\%), Eltern-/Familienarbeit (19\%), Pflege der Herkunftskultur(en) (19\%), Antidiskriminierungsarbeit (19\%), politische Interessenvertretung, Religion, Sport (jeweils ca. $15 \%$ ), Pflege/Unterricht der Herkunftssprache (13\%), Entwicklungszusammenarbeit (12\%), Übersetzungen (11\%), Seniorenarbeit (10\%), Gesundheit ( $8 \%$ ), Wissenschaft und Forschung (5\%), Arbeitsvermittlung (5\%), Umweltund Naturschutz (4\%), Andere (11\%) (Friedrichs et al. 2020: 23).
} 
mehr als gesellschaftspolitisch relevante und weniger als Mitgliederorganisationen (Priemer 2017: 2).

Die Untersuchung des SVR legt dar, dass rund 58 Prozent der MSO 100 Mitglieder, 50 Prozent weniger als 60 und 16 Prozent über 200 Mitglieder haben. Dabei ist hinsichtlich der Migrationsbezüge von einer vielfältigen Durchmischung der Mitgliederstruktur und nicht immer von der Existenz einer Migrationsbiografie auszugehen (Friedrichs et al. 2020: 18 f.). Im Durchschnitt besitzen drei Viertel der Mitglieder eine Zuwanderungsgeschichte, über die Hälfte (53\%) der Mitglieder sind weiblich und Frauen übernehmen in den MSO häufig Leitungspositionen (ebd.: 28). Die Autoren der Studie führen die hohe Vertretung von Frauen in den befragten MSO auf die häufig im sozialen und Bildungsbereich angesiedelten Tätigkeits- bzw. Engagementschwerpunkte der MSO zurïck, da sich in diesen - den Ergebnissen des Freiwilligensurveys 2014 zufolge - viele Frauen engagieren (ebd.). In Bezug auf die Angaben der Mitgliederzahlen seitens der MSO ist an dieser Stelle jedoch darauf hinzuweisen, dass nicht immer eindeutig ist, welche Personen die MSO im Rahmen der Erhebung konkret zu ihren Mitgliedern gerechnet und ob sie vielleicht auch Engagierte oder Nutzer ihrer Angebote dazugezählt haben, die ggf. keinen offiziellen Mitgliedschaftsstatus besitzen (ebd.: 20; II. 4.3.3).

Die Daten des ZiviZ-Surveys zeigen hingegen, dass jede zweite MSO weniger als 50 Mitglieder hat (Priemer 2017: 3). Zudem ist bei den - im Vergleich zu nicht-migrantischen Organisationen - befragten kleineren MSO seit 2012 ein positiver Trend bei der Entwicklung der Mitgliederzahlen zu verzeichnen (42\% vs. nicht-migrantische: $35 \%$ ). 45 Prozent der MSO berichten von steigenden Zahlen sich engagierender Personen (seit 2012), wohingegen lediglich 21 Prozent aller nicht-migrantischen Organisationen mehr Freiwillige gewinnen konnten. Mit äußerster Vorsicht sind die Befunde als Indiz zu deuten, dass MSO häufiger personellen Zuwachs bekommen als nicht-migrantische Organisationen.

Wenngleich Ehrenamtliche in der Arbeit der befragten MSO eine große Rolle spielen, zeigt die SVR-Studie, dass viele von ihnen auch mit bezahlten Beschäftigten arbeiten und sie somit durchaus auch professionell aufgestellt und nicht - wie häufig vermutet - strukturschwach sind. Lediglich 41 Prozent der untersuchten MSO verfügen nicht über bezahlte Mitarbeiter, was darauf schließen lässt, dass die MSO in den letzten Jahren einen „Professionalisierungsschub“ erfahren haben (Friedrichs et al. 2020: 45) ${ }^{12}$. Jedoch ist die Entlohnung von Arbeitskräften in den MSO auf sehr unterschiedliche Beschäftigungsformate zurückzuführen, sie fällt

\footnotetext{
${ }^{12}$ Im Vergleich zu den Daten des jüngsten ZiviZ-Surveys fallen die zu den bezahlten Beschäftigten ermittelten Zahlen hoch aus, denn dem ZiviZ-Survey zufolge verfügten 73 Prozent
} 
sehr vielfältig aus und bedeutet nicht automatisch, dass hauptamtliche Strukturen vorhanden sind (ebd.: $44 \mathrm{f}$.).

Der SVR-Studie zufolge arbeiten die meisten MSO (41\%) mit Honorarkräften, 37 Prozent der MSO verfügen über fest angestellte Voll- oder Teilzeitkräfte, 16 Prozent arbeiten ausschließlich mit Honorarkräften und 5 Prozent der migrantischen Vereinigungen werden von Minijobbern, Bundesfreiwilligen (,Bufdis ') oder Praktikanten unterstützt. Im Zusammenhang mit diesen Befunden ist jedoch zu bedenken, dass professionell aufgestellte MSO aufgrund ihrer personellen Ausstattung an Befragungen häufiger teilnehmen dürften als dies MSO tun, deren Arbeit ausschließlich von Engagierten getragen wird (ebd.; III. 5.3).

Wie andere gemeinnützige Vereinigungen der Zivilgesellschaft verfügen die MSO über geringe finanzielle Mittel bzw. Einnahmen. 38 Prozent der in der SVRStudie befragten MSO stehen jährlich 10.000 Euro und 13 Prozent 1.000 Euro pro Jahr zur Verfügung. Einzelne MSO (3\%) geben an, über eine Million Euro zu verfügen (ebd.: 69 f.). Dabei erheben 83 Prozent der MSO Mitgliedsbeiträge, die allerdings für die Finanzierung der Aktivitäten der MSO nicht ausreichen. Weitere Finanzierungsquellen stellen Spenden, Fördergelder, aber auch selbst erwirtschaftete Gelder dar (ebd.: 70). Über die Hälfte der MSO (52 \%) erhalten sowohl eine Förderung als auch Spenden, 17 Prozent bekommen ausschließlich finanzielle Zuwendungen und 15 Prozent lediglich Spenden. 15 Prozent beziehen weder Fördermittel noch Spenden. Im Jahr 2019 wurden 59 Prozent der befragten MSO durch die Kommune und 46 Prozent durch ein Bundesland gefördert, vom Bund haben 38 Prozent Gelder empfangen. 32 Prozent der MSO gaben für das Jahr 2019 an, durch Stiftungen finanziell unterstützt zu werden (ebd.: 70 ff.).

Auch die Ergebnisse des ZiviZ-Surveys zeigen, dass jeder zweiten MSO im Jahr 2015 maximal 10.000 Euro zur Verfügung standen, den meisten der befragten MSO jedoch viel weniger. Die Haupteinnahmequellen stellten zu jeweils 29 Prozent Mitgliedsbeiträge und - insbesondere bei religiösen MSO - Spenden dar. Von öffentlichen Fördermitteln profitierte jede dritte MSO. 41 Prozent der MSO erhielten nicht-finanzielle Unterstützungsleistungen von (zumeist) öffentlichen Einrichtungen in Form von Sachspenden oder kostenloser Nutzung von Räumlichkeiten. Damit profitieren die MSO in geringerem Umfang von dieser Art der Unterstützung als dies sämtliche Organisationen der Zivilgesellschaft mit insgesamt 59 Prozent tun (Priemer 2017: 3).

der MSO über keine bezahlten Beschäftigten und in 1 Prozent der MSO arbeitete ausschließlich hauptamtliches Personal (Priemer 2017: 3). Im gesamten Dritten Sektor können circa 28 Prozent aller gemeinnützigen Vereinigungen auf bezahlte Mitarbeiter zurückgreifen (Priemer/Schmidt 2019, zit. nach Friedrichs et al. 2020: 45). 
Die Studie des SVR stellt heraus, dass sich viele der MSO in (über-)regional und lokal aktiven Dachverbänden organisieren, wobei insbesondere die überregionalen Organisationen bedeutsam sind. 64 Prozent der befragten MSO gehören mindestens einem Verband an und jede fünfte MSO ist Mitglied in mehreren Verbänden, wobei es sich mit 50 Prozent zumeist um migrantische Dachverbände handelt. 23 Prozent der MSO haben eine Mitgliedschaft in Wohlfahrtsverbänden (v. a. dem Paritätischen Wohlfahrtsverband), die historisch zu begründen ist, und für 11 Prozent spielen religiöse Dachverbände eine Rolle. Jugend-, Eltern- und Sportverbände sind für die untersuchten MSO hingegen kaum relevant (Friedrichs et al. 2020: $54 \mathrm{f}$.).

Die SVR-Studie fördert zutage, dass sich die MSO mit anderen städtischen und zivilgesellschaftlichen Akteuren vernetzen. Am häufigsten kooperieren MSO mit Stadtverwaltungen (65\%), anderen gemeinnützigen (64\%) oder migrantischen Vereinen $(61 \%) .35$ Prozent der MSO arbeiten mit Kirchen und anderen Religionsgemeinschaften zusammen und 30 Prozent mit Wohlfahrtsverbänden sowie mit Bezirksverwaltungen und der Landespolitik (jeweils $27 \%$ ). Für knapp ein Viertel der MSO stellen Stiftungen, Hochschulen und informelle Initiativen (24\%) wichtige Kooperationspartner dar. 17 Prozent der MSO benennen bundespolitische Akteure und 15 Prozent Behörden und Organisationen im Ausland als Kooperationspartner (ebd.: 58 f.). Die mit den Kooperationen verfolgten Ziele betreffen Vernetzungen, die Verbesserung der Zugänge zu Infrastrukturen (Räumlichkeiten), den Austausch von Fachwissen, die (gemeinsame) Akquise von öffentlichen Geldern sowie die Erhöhung des Leistungsvermögens der MSO (ebd.: 60 ff.). Zwischen der Wahrnehmung eines Kooperationsverhältnisses als eine „Partnerschaft“ oder als eine „gleichberechtigte Zusammenarbeit" liegen jedoch teilweise starke Diskrepanzen; in Bezug auf die Stadtverwaltungen ist der Unterschied mit 46 Prozentpunkten am stärksten (Partnerschaft: $65 \%$ vs. gleichberechtigte Zusammenarbeit: $19 \%$ ). Bei den anderen genannten Kooperationspartnern beträgt der Unterschied zumeist 10 bis 20 Prozentpunkte (vgl. ebd.: 59).

Im Vergleich zur Studie des SVR-Forschungsbereichs hat auch die Untersuchung des ZiviZ-Surveys ergeben, dass Vernetzungen und Kooperationen mit kommunalen und zivilgesellschaftlichen Akteuren unter den MSO weit und stärker verbreitet sind als unter anderen zivilgesellschaftlichen Organisationen. Jede zweite MSO arbeitet mit einer anderen gemeinnützigen Vereinigung zusammen (Priemer/Schmidt 2018: 3). 22 Prozent der MSO sehen sich in einem kooperativen Verhältnis zu Kommunen und 21 Prozent stehen in einem Auftragsverhältnis zu einer Kommune. Charakter und Wert der Kooperationen als eine ,partnerschaftliche Zusammenarbeit" werden jedoch ebenfalls unterschiedlich beurteilt: 41 Prozent stimmen dem zu, 35 Prozent der MSO stehen dem ablehnend gegenüber 
(Priemer 2017: 2). Unter Bezugnahme auf andere Veröffentlichungen verweisen die Autoren des Survey-Berichtes darauf, dass „Kooperationen auf Augenhöhe“ erschwert seien, und zwar aufgrund fortbestehender Akzeptanz- und Anerkennungsprobleme, Vorbehalte und Anfeindungen gegenüber MSO (Priemer et al. 2017: 44; s. auch Hunger 2002; Hunger/Metzger 2011: 17 ff., 52 ff.; Nagel 2016: 86, 93; Friedrichs et al. 2020: 84 f.).

In Hinblick auf Unterstützungs- und Verbesserungsbedarfe wünschen sich auch 64 Prozent der im Rahmen der SVR-Studie erreichten MSO eine stärkere institutionelle und damit verlässliche Förderung, um die Verfügbarkeit von Personal und Infrastruktur dauerhaft sicherstellen zu können. 58 Prozent wünschen sich einen Abbau von Bürokratie und 51 Prozent Unterstützung bei der Antragstellung für den Bezug von Fördermitteln (Friedrichs et al. 2020: 79). ${ }^{13}$

Im Vergleich zu anderen Selbstorganisationen wünschen sich die MSO im Allgemeinen mehr staatliche Unterstützung. Fast 50 Prozent der im Rahmen des ZiviZ-Surveys befragten MSO reklamieren eine staatliche Finanzierung und jede zehnte MSO hält den Staat für die Erbringung der von ihnen erbrachten Leistungen zuständig. 42 Prozent der MSO befürworten Selbstfinanzierung sowie Eigenständigkeit und Unabhängigkeit der durch sie geleisteten Arbeiten (Priemer 2017: 3). Aufs Ganze gesehen ist dies ein Zeichen dafür, dass in Hinblick auf Rollenverhältnisse und Verantwortlichkeiten durchaus unterschiedliche Vorstellungen bestehen.

\subsection{Studien zu Migrantenselbstorganisationen mit dem Fokus auf türkisch-islamischen Verbänden}

Seit den 1980er Jahren intensivierte sich die wissenschaftliche Beschäftigung mit MSO in Deutschland. In der Studie von Raymond Breton (1964) über ethnische Gemeinschaften, Prozesse der Assimilation und Gruppenstabilisierung in der Stadt Montreal, sieht Ludger Pries einen wesentlichen Impuls und Anknüpfungspunkt für weitere Forschungen (Pries 2010a: 18). Bretons tendenziell negative Einschätzung selbstbestimmter eigenethnischer Strukturen (,,institutional completeness") in Hinblick auf das Zustandekommen interethnischer Beziehungen

\footnotetext{
${ }^{13}$ Weitere geäußerte Unterstützungs- und Verbesserungsbedarfe betreffen: mehr Unterstützung bei der Öffentlichkeitsarbeit (40\%), die Verbesserung der Kommunikation mit öffentlichen Stellen (36 \%) sowie des Zugangs zu Büros und Räumen (30 \%), die Gewährleistung des Vorhandenseins fester Ansprechpersonen in den Verwaltungen (25\%), die Vereinfachung der Amtssprache (17\%) sowie Verbesserungen beim Versicherungsschutz (13\%) (Friedrichs et al. 2020: 79).
} 
korrespondiert mit der später von Hartmut Esser vertretenen Hypothese, dass ethnische Binnenintegration für erfolgreiche Integration langfristig risikoreich sei (Esser 1986) (II. 4.1).

Eine Beschäftigung mit dem Forschungsstand zu MSO im deutschen Kontext sieht sich zunächst mit einer Eingrenzungsproblematik konfrontiert: Gemäß der im Rahmen dieser Arbeit breit angelegten Definition von MSO als einst von Zugewanderten und/oder deren Nachfahren in Deutschland gegründeten Selbstorganisationen wird unversehens ein weites, sehr heterogenes Spektrum an Zuwanderergruppen bzw. Organisationen zum Forschungsobjekt. Es bedarf daher der Einnahme einer Weitwinkelperspektive, um die Vielfalt der MSO zu berücksichtigen und damit der diesbezüglichen Forschungslandschaft gerecht zu werden. In Hinblick auf die dafür relevante Literatur steht man als wissenschaftlich forschende Person vor der Herausforderung einer breit anzulegenden Sichtungsarbeit, weil nicht alle wissenschaftlichen Titel, die infrage kommen, explizit das Stichwort „Migranten(selbst)organisation“ enthalten. Zudem besteht angesichts der Forschungslage die Tendenz zu einer Konzentration auf türkisch-islamische Organisationen, deren Dachverbände - als politische Interessenverbände - die meiste Aufmerksamkeit erhalten. ${ }^{14}$

Dem Islam und den (organisierten) Muslimen kommt als größte religiöse Minderheit in Deutschland in der Diskussion um Zuwanderung und Integration und aufgrund konfliktbeladener islambezogener Ereignisse das größte Interesse zu. Dies schlägt sich in der Forschung nieder. Das große Interesse an der Erforschung muslimischer Lebenswirklichkeit ist verknüpft mit wissenschaftlichen Konzepten und Theorien, die in den letzten Jahrzehnten und anhaltend Hochkonjunkturen erfahren (haben): Genannt seien hier zuvorderst die - auch politisch verwerteten - Konzepte zu Zivilgesellschaft, Sozialkapital sowie sozialer und systemischer Integration in ihren wechselseitigen Bezügen.

In den folgenden Abschnitten wird der Versuch unternommen, die zahlreichen Studien zu MSO in ihrer thematischen Vielfalt im Spektrum von Wissenschaft, Politik und Fachpraxis zu beleuchten. Dabei werden Zivilgesellschaft und Engagementbezug, wenngleich sie im Fokus vorliegender Arbeit stehen, nicht die

14 Überraschenderweise werden MSO trotz des erhöhten Interesses an ihrer wissenschaftlichen Erforschung und ihrer Anerkennung als wichtige integrationspolitische Akteure in einschlägigen Einführungs- und Übersichtswerken zur Migrations- und Organisationsforschung (z. B. Pries 2001; Allmendinger/Hinz 2002; Nuscheler 2004; Han 2005; Kalter 2008; Treibel 2008; Aigner 2017) oder zur politikwissenschaftlichen Verbändeforschung (Willems/Von Winter 2000; Sebaldt/Straßner 2004; Von Winter/Willems 2007) nicht gesondert thematisiert (siehe dazu auch die Ausführungen bei Pries/Sezgin 2010b: 7 f.). 
einzigen Kriterien der Betrachtung sein. Der Schwerpunkt liegt auf den türkischislamischen Organisationen bzw. Dachverbänden, die quantitativ den größten Teil der Studien ausmachen, und der synoptischen Darstellung der Geschichte ihrer Entwicklung und Erforschung in Deutschland. Zudem werden auch einschlägige jüngere Studien zu nicht-islamischen Organisationen berücksichtigt.

\section{0er bis 2000er Jahre}

Die Frage nach den integrativen Leistungen von MSO in der Aufnahmegesellschaft stand von Anfang an im Vordergrund. Sie stimulierte seit den 1980er Jahren zahlreiche empirische Studien, die in Form von einzelnen Monografien oder Aufsätzen veröffentlicht wurden. Dominant waren folgende Fragestellungen: Welche Motive sind bei Rückkehrabsichten entscheidend? Woran wird sich stärker orientiert, am Herkunfts- oder Aufnahmeland? Wonach richten sich die MSO im Rahmen von Willensbildungs- und Entscheidungsprozessen politisch aus? Über welche Mittel der Einflussnahme verfügen MSO, auf kommunaler und auf bundespolitischer Ebene?

In den Blick gerieten darüber hinaus die Organisationsstrukturen verschiedener ethnischer Minderheiten, ihre Tätigkeitsschwerpunkte, Kontakte sowie sozialen und kulturellen Funktionen. Die gesellschaftspolitischen Positionen und Aktivitäten wurden auch komparativ betrachtet. Im Vergleich mit anderen urbanen Ballungsgebieten und anderen Ländern waren unterschiedliche Rahmenbedingungen sowie integrative wie desintegrative Potenziale entsprechende Forschungsschwerpunkte (Breuer 1981; Schöneberg 1982; Jahn/Şen 1984; Haug 1985; Thränhardt 1985; Jahn 1986; Von Breitenbach 1986; Von Kodolitsch/Schuleri-Hartje 1987; Thränhardt 1989; Bommes 1991; Fijalkowski/Gillmeister 1997; Diehl/Urbahn 1998; Özcan 1989/1992; Puskeppeleit/Thränhardt 1990; Rütten 1996; Kößler 1997; Güngör 1999; Thränhardt/Hunger 2000; Diehl 2002).

Die einzelnen Studien befassten sich mit Selbstorganisationen griechischer, spanischer, jugoslawischer, italienischer, portugiesischer, koreanischer und weiterer Einwandergruppen $^{15}$. Ein deutlicher Schwerpunkt lag und liegt aber kontinuierlich auf der Untersuchung von türkischen und islamischen Organisationen bzw.

15 ISS 1980: Die griechischen Gemeinden in der BRD; Schlumm 1984a, b): Griechische Gastarbeiter und Organisationsform griechischer Gemeinden; Von Breitenbach 1979: Spanische Elternvereine; Alvares 1982: Spanische Selbsthilfegruppen und Organisationen in der Bundesrepublik; Romano-Garcia 1983: Der Bund der spanischen Elternvereine; Diaz 1987: Spanische Vereine in der BRD; Pusic 1980: Die Jugoslawen in der BRD; Büdel 1985: Die Selbstorganisationen der Jugoslawen in Dortmund; Hefner 1987: Zwei jugoslawische Vereine; Kuenzer 1987: Jugoslawisches Vereinsleben; Kammerer 1991: Italienische Immigrantenvereine in der BRD; Landwehr 1983, Mesquita 1980: Portugiesische Vereine in der BRD; Micksch 1986: Evangelische Ausländergemeinden; Thränhardt 1985: Türkische, griechische, spanische Selbstorganisationen im Vergleich; Yoo 1996, 1998: 
Dachverbänden ${ }^{16}$, wozu zum einen die dauerhafte Präsenz von Menschen islamischen Glaubens in Westdeutschland seit dem Anwerbestopp der Arbeitsmigranten im Jahr 1973 beigetragen hat, zum anderen aber auch die in Deutschland ausgetragenen Konflikte zwischen radikalen nationalistischen rechten und linken türkischen Gruppen.

Wesentliche Bestandsaufnahmen der MSO-Landschaft leisteten die Arbeiten von Rolf-D. Haug (1985), Jürgen Puskeppeleit und Dietrich Thränhardt (1990) sowie des Zentrums für Türkeistudien in Essen et al. (ZfTI 1995; ZfTI 1999; MASSKS 1999) ${ }^{17}$. Die Arbeiten spiegeln die Entwicklung wider, welche anfänglich von wohlfahrtsverbandlicher Betreuung der Einwanderergruppen ${ }^{18}$ geprägt war, später von

Interessenvertretung und Selbstorganisationen koreanischer Immigranten in Deutschland; Thränhardt/Dieregsweiler 1999, Soprat 2000: Polnische Selbstorganisationen in der BRD.

${ }^{16}$ Abdullah 1980: Drei muslimische Dachverbände in der Bundesrepublik Deutschland; Gesellschaft für Sozialforschung und Sozialplanung 1981: Türkische Organisationen in der BRD; Hoffmann et al. 1981: Graue Wölfe, Idealistenvereine und türkische Faschisten in Deutschland; Sezer/Thränhardt 1983, Sezer 1986: Türkische Organisationen in der BRD; Gitmez/Wilpert 1987: Soziale Organisation und Ethnizität türkischer Migranten in Berlin; Özak/Sezer 1987: Türkische Organisationen in der BRD; Binswanger/Sipahioğlu 1988: Türkisch-islamische Vereine; Nirumand/Bamdadan 1990: Islamische Gruppen und Fundamentalismus in der BRD; Özcan 1989/1992: Türkische Immigrantenvereine in der BRD (politische Organisationen und Orientierungen); Gür 1993: Türkisch-islamische Vereinigungen in der BRD; Schultze 1994: Die Bedeutung von Vereinen für die Identität junger Türken in Deutschland; Karakaşoğlu-Aydin 1996: Neuere Entwicklungen bei türkisch-islamischen Dachverbänden; Feindt-Riggers/Steinbach 1997: Aktuelle Bestandsaufnahme und Analyse islamischer Organisationen in Deutschland; Kenan 1997: Politische und Integrationsrelevanz türkischer Organisationen; Yalçın-Heckmann 1998: Risiken ethnischen Vereinslebens türkischer Migranten in Deutschland und Frankreich; Lemmen 1998, 1999: Türkisch-islamische Organisationen und muslimische Spitzenverbände (Islam- und Zentralrat) in Deutschland; Güngör 1999: (Des-)Integratives Potential türkischer Selbstorganisationen unter Berücksichtigung des stadtteilspezifischen Umfelds; Çetinkaya 2000a, b: Türkische Selbstorganisationen in Deutschland.

${ }^{17}$ Haug (1985): Initiativgruppen in der Ausländerarbeit und Selbstorganisation der Ausländer. Verbreitung, Bedeutung und Möglichkeiten; Puskeppeleit/Thränhardt (1990): Vom betreuten Ausländer zum gleichberechtigten Bürger; ZfTI (1995): Studien über islamische Organisationen der türkischen, marokkanischen, tunesischen und bosnischen Minderheiten in Hessen; ZfTI (1999): Bestandsaufnahme der Potentiale und Strukturen von Selbstorganisationen von Migrantinnen und Migranten türkischer, kurdischer, bosnischer und maghrebinischer Herkunft in Nordrhein-Westfalen bzw. MASSKS (1999): Selbstorganisationen von Migrantinnen und Migranten in NRW. Wissenschaftliche Bestandsaufnahme.

${ }^{18}$ Die Sozialberatung der Ausländer aus unterschiedlichen Herkunftsländern wurde zwischen den drei Wohlfahrtsverbänden aufgeteilt. Die Caritas war für die soziale Versorgung der katholischen Italiener, Spanier, Portugiesen und Jugoslawen zuständig. Die Diakonie war für die 
Emanzipation und Eigeninitiative der MSO, die Selbsthilfestrukturen auszubilden begannen und politische Mitwirkung beanspruchten.

Uwe Hunger (2002) hat herausgearbeitet, wie sich die anfänglich dominanten Typen des religiösen und des als Begegnungszentrum fungierenden ,Arbeitervereins“ zu Vereinen für Kultur, Sport und Bildung entwickelten, die sich aber auch mit wirtschaftlichen, politischen und sonstigen Anliegen der Zuwanderungsgruppen (Italiener, Spanier, Griechen, Türken und ehemaligen Jugoslawen) befassten. ${ }^{19}$

\section{Ab 2000er Jahre}

Was die Wirkungsrichtungen der MSO betrifft, ist seit den 2000er Jahren aufgrund des integrationspolitischen Paradigmenwechsels eine potenzialorientierte Betrachtungsweise zu registrieren. Zurückzuführen ist dies v. a. auf einen Wandel in der Wahrnehmung der MSO von vormals betreuten zu zunehmend eigenverantwortlichen und politisch handelnden Organisationen. Insbesondere die aufnahmegesellschaftlichen Institutionen begannen die Auffassung zu vertreten, dass den MSO mehr Verantwortung übertragen werden müsste (,Empowerment“) (Hunger 2002, 2004; Hunger/Candan 2009). Zudem werden die MSO fortan stärker in ihren transnationalen Bezügen (Pries/Sezgin 2010a, 2012; Sezgin 2009) und in thematisch ausdifferenzierten Perspektiven untersucht.

Dies hatte ein breites Spektrum an Forschungsergebnissen zur Folge, wodurch die enorme Vielfalt der zumeist herkunftshomogenen, zunehmend aber auch kulturell durchmischten MSO (Halm et al. 2012a: 59, 103; Priemer et al. 2017: 44; Friedrichs et al. 2020: 27; Halm et al. 2020: 121) und die unter den Einwanderungsgruppen sehr unterschiedlich ausgeprägte Affinität zur Selbstorganisation verdeutlicht werden konnten. ${ }^{20}$ Im Allgemeinen wird den MSO eine enorme Dynamik und Wandlungsfähigkeit attestiert, sie besäßen vielfältige Funktionen, übernähmen Unterstützungsleistungen für unterschiedliche Zielgruppen und seien durch einen ausgeprägten Selbsthilfecharakter gekennzeichnet (Pries 2010a, 2013a, b; Gaitanides 2003; Oswald 2007: 123; Weiss/Thränhardt 2005a; Halm/Sauer 2006: 21; II. 4.1).

griechischen und die Arbeiterwohlfahrt (AWO) für alle nichtchristlichen, v. a. die türkischen und jugoslawische Migranten, verantwortlich (Puskeppeleit/Thränhardt 1990: 47 ff.).

${ }^{19}$ Siehe für einen kompakten Überblick zur Entwicklungsgeschichte von MSO in Deutschland auch: Mualem Sultan et al. 2019: 6-12.

${ }^{20}$ Als allgemeiner Befund lässt sich diesbezüglich z. B. das im Vergleich zu türkeistämmigen Menschen bei polnischen Gruppierungen deutlich geringer ausgeprägte Bedürfnis nach Selbstorganisierung nennen (Sopart 2000; Nowosielski 2012; Halm et al. 2012b). 
Wie im vorigen Kapitel bereits verdeutlicht wurde, sind die MSO regional disparat verteilt, was zum einen mit unterschiedlichen Zuwanderungsdichten zusammenhängt, zum anderen mit den jeweiligen politischen Opportunitätsstrukturen auf nationaler, landes- und kommunalpolitischer Ebene erklärt wird (Vermeulen 2006; Kortmann 2011; Van Houte et al. 2013).

Im Folgenden werden einige jüngere, in unterschiedlichen Fachdisziplinen verortete Studien und deren Ergebnisse skizziert, die exemplarisch die Vielfalt der Themen und methodischen Ansätze verdeutlichen sollen. Wenngleich sie keinen Anspruch auf Repräsentativität und Vollständigkeit haben, dürften sie in unterschiedlichem Ausmaß für alle MSO Relevanz besitzen.

\section{Jüngere Studien unterschiedlicher Fachdisziplinen}

Einige komparativ angelegte politikwissenschaftliche Studien setzen auf staatlicher Ebene an und vergleichen Deutschland und die Niederlande in Hinblick auf die jeweiligen Verhältnisse und Gelegenheitsstrukturen und deren Auswirkungen auf die Entwicklung spezifischer Profile der näher untersuchten MSO (Vermeulen 2006; Musch 2011; Kortmann 2011). So analysiert Matthias Kortmann (2011) in seiner vergleichenden Studie, inwieweit nationale Kontextbedingungen (,,political opportunity structures") in Deutschland bzw. in den Niederlanden die Ausrichtungen und strategischen Handlungsweisen von vorrangig türkisch-muslimischen Dachverbänden beeinflussen. Auf Basis der Analyse des Selbst- und Integrationsverständnisses der MSO kann Kortmann nachweisen, dass sich die MSO in Deutschland in erster Linie - aufgrund des in Deutschland gegebenen Religionsverfassungsrechts - als privilegierte Religionsgemeinschaften verstehen, die sich transnational verpflichtet sehen und zugleich auf Akkulturation im Aufnahmeland ausgerichtet sind. Hingegen profitierten die MSO in den Niederlanden aufgrund ihres Status als soziale, ethnisch-kulturelle und weniger religiöse Organisationen von der niederländischen Minderheitenpolitik und insistierten im Rahmen der Tradition des Multikulturalismus auf die Bewahrung ihrer Herkunftsidentität.

Weitere politikwissenschaftliche Analysen fokussieren politische Gelegenheitsstrukturen und die damit einhergehenden Zugangschancen für Einwandererverbände in Deutschland (Leinberger 2006; Blätte 2014). Andreas Blätte (2014) analysiert die Ressourcen, Potenziale und Handlungslogiken von unterschiedlichen (nicht-)religiösen Einwandererverbänden sowie ihre (in-)formalen Zugangsmöglichkeiten zu politischen Entscheidungsprozessen innerhalb der deutschen Migrations- und Integrationspolitik zwischen 1998 und 2006. Er zeigt auf, dass die neue bundesrepublikanische Integrations- und Islampolitik, die auf Kooperation setzt, eine grundsätzliche Veränderung der politischen Gelegenheitsstrukturen 
bewirkt hat, welche die islamischen Verbände begünstigt und eine Schieflage habe entstehen lassen, die v. a. durch eine vermehrte Repräsentation von anderen Einwanderergruppen in Parteien, Parlamenten und Regierungsämtern kompensiert werden könnte (ebd.: 81, 250).

Neben politikwissenschaftlichen Analysen sind in den letzten Jahren sozialwissenschaftliche Studien zu unterschiedlichen Themen durchgeführt worden: zu Vereinen von Jugendlichen mit Migrationshintergrund (kurz: VJM) (Jagusch 2011), zu zivilgesellschaftlichen Anliegen und Beteiligungsmöglichkeiten von MSO (Josten 2012) sowie zu Frauen mit Zuwanderungsgeschichte und ihrer Situation in Vereinen (Ilgün-Birhimeoğlu 2017).

Die Arbeit von Birgit Jagusch (2011) zeigt auf, dass sich Vereine von Jugendlichen mit Migrationshintergrund ${ }^{21}$ in ihrem Streben nach Wertschätzung, Akzeptanz sowie nach Verarbeitung und Bewältigung erfahrener Ausgrenzung und Diskriminierung eigene Räume von Anerkennung und Empowerment schaffen. Durch die Existenz dieser Räume würden Mehrfachzugehörigkeiten zugelassen, Essentialisierungen vermieden und eine selbstbewusste (zivil-)gesellschaftliche Partizipation ermöglicht (Jagusch 2011: 423-430).

Anhand von Dokumentenanalysen registriert auch Daniel Josten (2012) bei ausgewählten Migrantengruppen ${ }^{22}$ ein Streben nach Anerkennung, Einflussnahme und Ausgleich von Exklusionserfahrungen sowie Varianten (zivil-)gesellschaftlichen Engagements und politischer Partizipation, die sich im Kontext gegebener Spielräume, Machtverteilungen und gesellschaftlicher Platzierungen realisierten (Jagusch 2011: 206-209).

Emra Ilgün-Birhimeoğlu (2017) untersucht in ihrer multimethodischen Studie die Teilhabechancen von Frauen mit Migrationshintergrund in migrantischen und ,deutschen“ Vereinen. Sie identifiziert strukturell verankerte Ausgrenzungsund Abwertungsmechanismen und eine doppelte Diskriminierung: aufgrund ihres Geschlechts in Migrantenvereinen und aufgrund ihrer Zuwanderungsgeschichte in „,deutschen“ Vereinen. Der kontinuierliche Vergleich mit der deutschen Frau als „Norm“ (Ilgün-Birhimeoğlu 2017: 240) resultiere in einer defizitbezogenen Betrachtungsweise und ungleichen Zugängen zu freiwilligem Engagement (ebd.: 239-244).

\footnotetext{
${ }^{21}$ Bund der Alevitischen Jugendlichen in Deutschland (BDAJ); Jugendverband der Föderation Demokratischer Arbeitervereine e. V. (DIDF-Jugend); Deutsche Jugend aus Russland (DJR).

${ }^{22}$ Föderation demokratischer Arbeitervereine e. V. (DIDF); PHOENIX-Köln e. V.: ein Verein von russischsprachigen Immigranten; Gruppen von Illegalisierten.
} 
Zwei weitere jüngere Untersuchungen haben einen dezidierten Religionsbezug gewählt. Sie fokussieren Motivstrukturen von in christlichen und muslimischen Verbänden freiwillig Engagierten (Klöckner 2016) sowie die Bedeutung von Religion für Partizipationsprozesse von religiösen migrantischen Selbstorganisationen im binationalen Vergleich (Schader 2017). So zeigt Miriam Schader (2017) am Beispiel von religiösen Selbstorganisationen von Zuwanderern aus Subsahara-Afrika in Berlin und Paris, dass diese ihre Religionszugehörigkeit in einem (neuen) Aufenthaltsland unter bestimmten Umständen (!) als Ressource für politische Beteiligung nutzen können. Die Autorin weist nach, dass Religion für christliche Organisationen als mobilisierende Ressource fungieren kann, und zwar in höherem Maße als für muslimische, nicht-religiöse und säkulare Organisationen (ebd.: 224). Deutlich werde, dass die MSO die Religion in zweifacher Hinsicht nutzen können: einerseits als ein geteiltes, nach innen einheitsstiftendes Identitätsmerkmal und andererseits als ein nach außen, in die Gesellschaft hineinwirkender Faktor, der Legitimität verschafft (ebd.: 220). Ausschlaggebend sei dabei, wie die jeweilige Religionszugehörigkeit in der Aufnahmegesellschaft angesehen ist (die christliche ist höher angesehen als die islamische) und ob die religiöse Selbstbeschreibung einer MSO als für ihre Partizipation legitim wahrgenommen wird (was in Deutschland besser funktioniere als in Frankreich). Die Bezugnahme auf Religion könne sich als geeignete Ressource für die Überwindung von mit ethnischer Zugehörigkeit (Subsahara-Afrika) verbundenen aufnahmegesellschaftlichen Zuschreibungen erweisen.

Jennifer Klöckner (2016) hat mit interdisziplinären theoretischen Ansätzen die Engagementmotive von in türkisch-islamischen sowie in Wohlfahrtsverbänden freiwillig Mitarbeitenden unter besonderer Berücksichtigung der Bedeutung von Religionszugehörigkeiten und Religiosität untersucht. Die qualitativ und quantitativ angelegte Studie fördert komplexe Zusammenhänge zutage und weist nach, dass religiöse Motive auf unmittelbare und vielfältige Weise mit sozialen, nutzenorientierten und weniger altruistischen Motiven verknüpft sind. Zudem zeigt die Erhebung, dass die türkisch-islamischen Vereine mit ihrer je spezifischen weltanschaulichen Ausprägung hinsichtlich der Gewinnung von Engagierten und deren persönlichen Motiven ähnliche Wirkungen zeigen und somit vergleichbar sind (Klöckner 2016: 434 ff.). Anhand der Aussagen der Befragten seien keine ,parallelgesellschaftlichen Tendenzen“ (ebd.: 443) zu registrieren, sondern vielmehr der Wunsch nach einer weiteren Annäherung an institutionalisierte Strukturen entsprechend dem Vorbild der Wohlfahrtsverbände. 


\section{Wachsendes politisches Interesse an MSO und interkulturellen Öffnungspro- zessen}

Im Zuge einer auf Bundes-, Landes- und kommunaler Ebene zunehmend wichtiger werdenden Engagementpolitik und Engagementförderung geraten das freiwillige Engagement von Menschen mit Migrationshintergrund, die zivilgesellschaftlichen Aktivitäten und Angebote der MSO sowie deren gesamtgesellschaftliche Einbindung verstärkt in den Blick (Huth 2007, 2011a, b, 2013; Groß et al. 2017; BMFSFJ 2017: 192-221). In diesem Zusammenhang ist insgesamt auf Wissensdefizite und Forschungsdesiderate im Bereich des Engagements von Menschen mit Zuwanderungsgeschichte und ihren Organisationen sowie auf einen Mangel an repräsentativen Erhebungen zu unterschiedlichen Einwanderergruppen zu verweisen (vgl. BMFSFJ 2017: 491). Angesichts der mangelhaften Forschungslage ist zukünftig die Durchführung von mehr sozialwissenschaftlichen Studien geboten.

Mit dem hohen Interesse verknüpft ist die wissenschaftliche Eruierung von Kooperationsmöglichkeiten mit (islamischen) MSO (Schmid et al. 2008; Rosenow/Kortmann 2010; Schröder 2014). Broschüren und Praxisleitfäden haben die Arbeit entsprechender Initiativen und Netzwerke zur Engagementförderung sowie erfolgreiche Kooperationen dokumentiert. ${ }^{23}$ Darüber hinaus kommt entwicklungspolitischen Initiativen und Projekten in den Heimatländern eine erhöhte Bedeutung zu, denn im Rahmen der deutschen Entwicklungszusammenarbeit soll mithilfe der MSO in Deutschland an der Verbesserung der Lebenssituation der Menschen in den Herkunftsländern gearbeitet werden (Haase/Müller 2012; CIM et al. 2013; Engagement Global 2014a, b; Winterhagen 2015; Friedrichs et al. 2020: 24 f.; BMZ $2021^{24}$ ).

Weitere Schwerpunkte lagen bei politischerseits in Auftrag gegebenen Forschungsarbeiten auf der Sondierung von bundesweit und regional tätigen (religiösen) Migranten(dach)organisationen, sofern diese besondere fachliche und integrationsrelevante Ausrichtungen haben (IntB 2011), auf Aktivitäten von Frauen innerhalb von Migrantinnenselbstorganisationen (Reinecke et al. 2010, 2011) sowie auf türkeistämmigen Personen in organisierten Kontexten (Halm/Sauer 2005; Sauer 2011).

Wie bereits erwähnt wurde, sind Kooperationen zwischen migrantischen und nicht-migrantischen Organisationen gesellschaftspolitisch wichtig geworden.

${ }^{23}$ MOZAIK 2009, 2016; BBE 2007, 2008, 2009, 2010; für NRW u. a.: KI Bielefeld 2016, 2017; Stadt Herne - Der Oberbürgermeister 2013; mit einem Schwerpunkt auf Arbeitsmarktintegration: MOZAIK 2006a, b, c, 2007, 2011, 2014.

${ }^{24}$ URL: https://www.bmz.de/de/entwicklungspolitik/migration/engagement-von-migranten22054 (letzter Abruf am 03.04.2021). 
Dementsprechend arbeiten professionelle Wohlfahrtsverbände daran, Kompetenzen auf MSO zu übertragen (DCV 2010, 2011; DPWV 2011; Schmidt 2012; Toker 2013). In diesem Zusammenhang sind interkulturelle Öffnungsprozesse bedeutsam geworden $^{25}$.

Hinsichtlich der Kooperationsförderung ist an dieser Stelle auf die im Auftrag des Bundesamtes für Migration und Flüchtlinge (BAMF) von Uwe Hunger und Stefan Metzger (2011) durchgeführte qualitative Studie Kooperation mit Migrantenorganisationen zu erwähnen. Aufgrund der gewählten Analyseebenen und der sondierten (Miss-)Erfolgsfaktoren erweist sie sich für das Forschungsinteresse der vorliegenden Arbeit als relevant. Die beiden Autoren haben in ihrer Studie sogenannte Tandemprojekte untersucht, bei denen es sich um in vielen Bundesländern geförderte Kooperationen zwischen etablierten, zumeist einheimischen Institutionen der Aufnahmegesellschaft sowie noch unerfahrenen MSO zwecks deren Qualifizierung handelt.

Anhand von drei Analyseebenen - internen, externen und relationalen Bedingungen - haben die Autoren wesentliche Einflussfaktoren identifiziert, die sich fallspezifisch als Förder- und Hemmfaktoren für Kooperationen erweisen können (Hunger/Metzger 2011: 52-80; vgl. auch Hunger/Metzger 2013b: 85-106; Nagel 2015b: 27-31; Schumacher/Huth 2013). Deutlich wird, dass diese Tandemprojekte keine Selbstläufer sind, sondern dass hinsichtlich der partnerschaftlichen Zusammenarbeit durchaus Barrieren bestehen. $\mathrm{Zu}$ nennen sind $\mathrm{u}$. a. potenziell bestehende Macht- und Wissensasymmetrien, wahrgenommene Konkurrenzsituationen um Fördergelder, Differenzen in puncto Professionalität und Erfahrungswissen, strukturelle Schwächen der MSO, Sprachschwierigkeiten, gegenseitige Fremdheitsgefühle und Vorurteile, paternalistische Haltungen gegenüber den Migrantenvereinen sowie Gefühle des Instrumentalisiert-Werdens aufseiten der MSO (siehe dazu auch BMFSFJ 2017: 211-213; Klie 2018: 475 ff.). Die Autoren resümieren, dass es daher für die erfolgreiche Durchführung solcher Tandems vorab einer grundlegenden Klärung des Verhältnisses zwischen den für die Zusammenarbeit vorgesehenen Organisationen bedarf.

\section{Islamische MSO in Deutschland: Entwicklungsgeschichte und Forschungs- schwerpunkte}

Aufgrund der Anwesenheit großer Gruppen von türkeistämmigen Muslimen, die gegenwärtig die größte Gruppe der Menschen mit Zuwanderungsgeschichte und

${ }^{25}$ Aus der Vielzahl der Literatur exemplarisch: Grünhage-Monetti 2006; DRK 2007; Curvello 2010; Handschuck/Schröer 2012; Schumacher/Huth 2013; Hunger/Metzger 2013; MOZAIK 2015; Jähnichen et al. 2016; Cortés Núñez/Kofli 2017. 
Minderheitenstatus in Deutschland darstellen (Destatis 2019a; Brinkmann 2016: 151; Haug et al. 2009: 303 f.), und angesichts der seit der Nachkriegszeit fortschreitenden Institutionalisierung des Islam in Deutschland ist im Folgenden ausführlicher auf empirische Studien zu islamisch-türkischen Gemeinden einzugehen. Auf Basis einer eigenen Auswertung verfügbarer Datensätze wurde bereits dargelegt, dass die türkisch-islamischen Vereine bzw. Dachverbände den größten Anteil der für NRW ermittelten Anzahl von MSO ausmachen. Daher sollen eine kurze schlaglichtartige Rekapitulation der verwickelten Gründungsgeschichte und eine kurze Charakterisierung der Dachverbände erfolgen. Die in den Studien gewählten thematischen Akzentsetzungen und Zugänge sind immer auch vor dem Hintergrund islambezogener weltpolitischer Vorgänge sowie komplexer, aufeinander bezogener Ereignisse in der Türkei und in Deutschland zu betrachten.

Werner Schiffauer unterteilt die Geschichte des türkischen Islam und seiner Konsolidierung in Deutschland in eine erste Phase von den 1970er bis Mitte der 1980er Jahre und eine zweite Phase ab Mitte der 1980er Jahre. Die 1970er Jahre waren gekennzeichnet durch die Aussicht auf einen nicht nur vorübergehenden, sondern längeren Verbleib in Deutschland. Daraus resultierten Gründungen der sogenannten „Hinterhofmoscheen“ bottom up durch aktive Gläubige, die in der Türkei häufig Mitglieder von teilweise illegal eingestuften islamisch-politischen Organisationen, Parteien oder Bruderschaften waren (Schiffauer 2000: 17 f.; Tezcan 2016: 158 f.). Zu letzteren gehörten die faschistische Partei und Organisation der Nationalistischen Bewegung (Milliyetçi Hareket Partisi - MHP), die auch unter der Selbstbezeichnung „Graue Wölfe“ (türkisch: Bozkurtçular) firmiert, die sufistischen Süleymancı (Anhänger Suleymans) und die Nurcus (,Anhänger des Lichts“), die dem 1973 in Köln gegründeten sunnitischen Verband der Islamischen Kulturzentren (VIKZ) zuzurechnen sind.

Die 1976 in Köln gegründete Vorläuferorganisation der Islamischen Gemeinschaft Millî Görüş (IGMG) hat eine lange und komplizierte Entstehungsgeschichte. Sie ist mit der islamistischen Partei Necmettin Erbakans und einer heftigen internen Auseinandersetzung um die politische Ausrichtung im Jahr 1983 verknüpft. Diese führte zur Abspaltung einer zwei Drittel Mehrheit der radikalislamistischen Kaplan-Anhänger, die sich im Verband der islamischen Vereine und Gemeinden e. V. (ICCB) - dem späteren Kalifatstaat - versammelten.

Die Türkisch-Islamische Union der Anstalt für Religion e. V. (türkisch: Diyanet İşleri Türk İslam Birliği - DİTİB) wurde erst 1984 in Köln und damit bedeutend später gegründet als alle anderen türkisch-islamischen Verbände. Die Gründung geht auf eine Initiative des 1924 in Ankara eingerichteten Amts für religiöse Angelegenheiten (Diyanet İşleri Başkanlığı; kurz: Diyanet) zurück und hatte zum Ziel, in Deutschland für die türkeistämmigen Muslime einen laizistisch geprägten Islam 
zu etablieren ${ }^{26}$. Zu erwähnen ist ferner die 1989 gegründete Alevitische Gemeinde Deutschland e. V. (Almanya Alevi Birlikleri Federasyonu - AABF), der wichtigste alevitische Dachverband. Ob und inwieweit sich Angehörige des Alevitentums der islamischen Gemeinschaft zugehörig fühlen oder sich als eigenständige Religionsgemeinschaft verstehen (sollten), ist ein inneralevitisch wie innermuslimisch kontrovers diskutiertes Thema. ${ }^{27}$ Alle Verbandszentralen siedelten sich in Köln (NRW) an (siehe zur Geschichte der türkisch-islamischen Verbände: Lemmen 2002; Wunn 2007; Lemmen 2017).

Die Etablierung der islamischen Verbände und die muslimische Präsenz und Sichtbarkeit im öffentlichen Raum stimulierten ab den 1980er Jahren zahlreiche wissenschaftliche Studien, die primär in der qualitativen Feldforschung zu verorten sind und das soziale Handeln muslimischer Individuen in organisierten Kontexten analysieren (s. dazu auch Fußnote 16). In den 2000er Jahren erschienen sodann einige islamwissenschaftliche Publikationen, die auf Basis von Dokumentenanalysen islamische Gruppierungen untersuchen (z. B. Heine 2000; Lemmen 2002; Kreitmeir 2002). Quantitative Studien zu islamischen Organisationen sind erst später populär geworden; ihre Ergebnisse werden im Textverlauf später referiert.

Die deskriptiven, auch normativen und politischen Studien zu islamischen Gruppen und einzelnen Gemeinschaften sind von Anfang an mit den Fragen nach der Kompatibilität des Islam mit der Moderne und seiner Integrierbarkeit verknüpft (Tezcan 2003). Integrationsdefizite in den 1970er und 1980er Jahren waren durch bürgerkriegsähnliche Zustände in der Türkei (1975-1979) sowie durch die Links-Rechts-Konfliktlinie zwischen gegnerischen türkischen Gruppen aus dem nationalistischen rechten und linken Spektrum geprägt ${ }^{28}$. Erst in den 1990er Jahren endete das Konflikt-Szenario, um angesichts einer international erstarkenden islamischen Radikalisierung in eine Fundamentalismusforschung umzuschlagen (Tezcan 2003: 241). Parallel zu dieser Entwicklung werden islamische Verbände zunehmend zu Repräsentanten der in Deutschland lebenden Muslime und zu Ansprechpartnern für die deutsche Politik in islambezogenen und integrationspolitischen Belangen

\footnotetext{
${ }^{26}$ DİTIB-Gemeinden werden im Rahmen der vorliegenden Arbeit als MSO verstanden, wenngleich sie dem Einfluss und der Kontrolle der türkischen Religionsbehörde unterliegen und als Diyanet-Außenvertretungen oder -Ableger betrachtet werden (Gorzewski 2015: 73 ff.; s. auch die Einleitung).

${ }^{27}$ Zentrale Aspekte zur Situation, zum Selbstverständnis sowie zu den Anerkennungsbemühungen der Aleviten in Deutschland geben die Sammelbände von Martin Sökefeld (2008) und Friedmann Eißler (2017).

${ }^{28}$ Einen guten Überblick zu den türkischen politischen Organisationen geben u. a. Sezer (1986), Gitmez/Wilpert (1987) und Özak/Sezer (1987).
} 
(Einleitung). Anhand dieser Szenarien lässt sich der ambivalente Charakter islamischer Organisationen kennzeichnen: Die gegenüberstehenden Pole reichen von einem ihnen innewohnenden antidemokratischen Gefährdungspotenzial auf der einen und dem genuinen Wunsch und Bedarf, Bestandteil der deutschen Gesellschaft zu werden auf der anderen Seite. Einigen islamischen Vereinen werden fortan auch Verschleierungstaktiken und Doppelstrategien vorgeworfen: Das Bekenntnis zum Grundgesetz nach außen widerspreche den nach innen vertretenen demokratie- und integrationsfeindlichen Positionen (u. a. Schiffauer 2004, 2005; vgl. auch Tezcan 2016: 163; Nds. LT 2012: $111 \mathrm{ff}$.).

Die Ambivalenzen und Konfliktpotenziale werden sich langfristig in der wissenschaftlichen Forschung und in den öffentlichen Debatten der jüngsten Gegenwart widerspiegeln. Flankiert werden diese Perspektiven von der Analyse von innerhalb islamischer Gruppen zu registrierenden Prozessen bzw. Fähigkeiten zur Transformation. Diese werden forciert durch die Einflussnahme und andere Bedarfe von jüngeren in Deutschland sozialisierten Generationen bzw. Mitgliedern, die Veränderungen in der Vereinsarbeit einleiten. Wandlungsprozesse werden zudem auf die Folgen gesellschaftlichen Anpassungsdrucks und wechselseitiger Zuschreibungsprozesse in ihrer Relevanz für die Produktion und Konstruktion islamischer Subjekte zurückgeführt, die die Forschung mit erhöhter Sensibilität zur Kenntnis nimmt (Tezcan 2003: 242).

Vor diesem Hintergrund untersuchten einzelne qualitative Studien in Form von Monografien islamische Gemeinschaften wie die radikalislamische KaplanGemeinde (Schiffauer 2000), den VIKZ (Jonker 2002) und die IGMG (Schiffauer 2010). Im Fokus stehen kollektive Praktiken, politische Ideen, Interessen und religiöse Weltbilder unter Berücksichtigung historischer und politischer Entwicklungen sowie des Einflusses von Einzelpersonen und der spezifischen Situation in der Diaspora. Immer präsent ist dabei das komplexe Spannungsfeld zwischen Türkeibindung einerseits und vielfältigen Öffnungsprozessen gegenüber der deutschen Gesellschaft andererseits. Dass dabei nicht von unilinearen Verläufen und einer Binnenhomogenität islamischer Gemeinden auszugehen ist, zeigen paradigmatisch die Studien von Werner Schiffauer $(2000,2010)$ und Gerdien Jonker (2002). So zeigt Jonker anhand ihrer Studie zum VIKZ, dass bereits eingeschlagenen Öffnungsprozessen der Gemeinde in Deutschland infolge politischer Führungswechsel in der Türkei wiederum Abschottungen folgen können. Und Schiffauer (2004, 2005, 2010) registriert einen aktiv vorangetriebenen Generationenwechsel und transformative Impulse innerhalb der IGMG, deren Anhänger sowohl islamistische als auch antiislamistische, wertekonservative islamische Positionen vertreten.

Die soziohistorischen Analysen spiegeln den Stellenwert von Moscheevereinen sowohl als religiöse als auch politisierte und politisierende Institutionen 
wider, deren Verbindungen zu Parteien und Mutterorganisationen von essenzieller Bedeutung für Positionierungen und Verhaltensformen ihrer Mitglieder im deutschen Kontext sind. Im Zuge der Debatten der Institutionalisierung des Islam in Deutschland befinden sich die türkisch-sunnitischen Verbände hinsichtlich ihrer Selbstbehauptung, Interessendurchsetzungen und dem dafür notwendigen Gewinn von Mitgliedern und Legitimität zudem in einer Konkurrenzsituation (Rosenow 2010; Rosenow-Williams 2012: 48, 67).

Das Bedürfnis nach Anerkennung als legitime Repräsentanten der Muslime und der Anspruch auf verfassungsrechtlich garantierte rechtliche Gleichstellung stärkt die Positionen der islamischen Verbände. Zudem werden muslimische Akteure aufgrund ihrer wichtigen Rolle als Ansprechpartner und Träger von integrationspolitischen Maßnahmen mit guten Zugängen zur muslimischen Community und zwecks Entschärfung extremistischer Gefahren gezielt zu Kommunikationspartnern der deutschen Politik (Azzaoui 2011; Tezcan 2016: 175 f.). Aktuell bleibt dabei die Frage, welche Tendenzen die vorherrschenden sind: die Entwicklung der Vereine und Verbände zu „Integrationsbrücken“ oder „Parallelgesellschaften“. Auf anhaltendes Forschungsinteresse stoßen diesbezüglich Organisationen wie DİTİB und Millî Görüss (Rosenow-Williams 2012; Gorzewski 2015; Beilschmidt 2016), die hinsichtlich ihrer Eigenschaften als politische Organisationen und religiöse Gemeinschaften eine Zwitterstellung einnehmen. Kontinuierlich kontrovers wird insbesondere die Abhängigkeit von DİTIB von der türkischen Religionsbehörde Diyanet sowie der (autokratischen) Politik Recep Tayyip Erdoğans debattiert. Die Diskussion erhält immer wieder neuen Aufwind durch politische Ereignisse in der Türkei und in Deutschland, wie etwa dem blutigen Putschversuch von Teilen des türkischen Militärs im Juli 2016, für den die türkische Regierung die GülenBewegung verantwortlich macht, und der 2016/17 in Deutschland zur sogenannten DİTİB-Spitzelaffäre ${ }^{29}$ führte (MDI 2018: 12). Die Verbindungen von DİTİB zur türkischen Religionsbehörde bzw. zum türkischen Staat provozieren fortwährend Fragen nach den tatsächlichen Zielsetzungen und der Legitimität des Status von DITIB als Kooperationspartner des deutschen Staates. Dabei spielt auch die Loyalität der Mitglieder gegenüber dem türkischen Staat und der Wunsch der Bewahrung der nationalen Identität eine bedeutende Rolle.

Empirisch fundierte Überlegungen zu der virulenten Frage, ob ein mentales und diskursives Sich-Einlassen auf deutsche Verhältnisse eine Abnabelung vom türkischen Kontext ermöglichen wird, fielen und fallen unterschiedlich aus. Während

${ }^{29}$ DİTİB-Imamen soll seitens der türkischen Religionsbehörde Diyanet der Auftrag gegeben worden sein, in Moscheen Informationen über Anhänger der Gülen-Bewegung zu sammeln und diese an die Behörde weiterzuleiten. 
beispielsweise Günter Seufert (1999a) vor der Jahrtausendwende die Möglichkeit einer öffentlichen Anschlussfähigkeit und eines Wandels durch Abkopplung von der türkischen Politik für DİTİB verneint, sieht er die Wahrscheinlichkeit einer Transformation und Abnabelung bei der IGMG (1999b) tendenziell eher gegeben (vgl. hierzu auch Schiffauer 2004, 2005).

Kerstin Rosenow-Williams (2012) kommt in ihrer Untersuchung islamischer Verbände zu dem Ergebnis, dass sich DITTIB in einem Balanceakt befinde, in einem „Spagat“ zwischen Herkunfts- und Aufnahmeland. Zugleich zeige sich aber das starke Bedürfnis, auf in Deutschland bestehende politische Erwartungen einzugehen bzw. sich ihnen anzupassen (Rosenow-Williams 2012: 460). Für die IGMG macht Rosenow-Williams eine Neupositionierung bei einer zugleich deutlich werdenden internen Spaltung der Organisation aus (ebd.: 461), wozu schlechtes Image und anhaltende Überwachung der Organisation durch den Verfassungsschutz beitrügen. Zudem begünstige die interne Kritik an der Marginalisierung der Organisation das Protestverhalten der IGMG (ebd.: 462). Es sei mithin kein Fall einer vollständigen, sondern lediglich einer partiellen Anpassung zu beobachten, und zwar auf der Ebene der Außenbeziehungen in Bezug auf allgemeine Fragen der Sicherheit und Zusammenarbeit (ebd.).

Andreas Gorzewski verweist hingegen auf erhebliche Veränderungen und einen nicht aufhaltbaren Wandel von DİTiB: „Insgesamt ist zu erwarten, dass sich die inhaltliche und institutionelle Ausrichtung der DİTIB auf Staat und Gesellschaft in Deutschland weiter fortsetzen wird" (Gorzewski 2015: 325). Dieser Prozess wurde durch die Anerkennung als Kooperationspartner für den Religionsunterricht und die Lehre an Universitäten begünstigt. Die Empfehlung Gorzewskis, dass der Wunsch nach Identitätsbewahrung und ,die wichtige Frage nach den Verbindungen zu Ankara [...] in den öffentlichen Debatten über die DİTİB und die Etablierung islamischer Organisationen in Deutschland nicht ausgeblendet werden“" (ebd.: 324) sollten, mutet angesichts jüngster konfliktgeladener Entwicklungen zwischen der Türkei und Deutschland, insbesondere in Hinblick auf die Aktualität der personellen und organisatorischen Abhängigkeit von DİTİB von ihrer Mutterorganisation anachronistisch an. Hier zeigt sich exemplarisch, dass wissenschaftliche Prognosen von Dynamiken politischer Entwicklungen überholt werden und mithin permanent revisionsbedürftig sind. 
Parallel zu diesen in Monografien und Sammelbänden ${ }^{30}$ thematisierten Entwicklungstendenzen sind islamische Individuen und Gruppierungen aufgrund des islamisch-fundamentalistischen Phänomens des Salafismus wie auch des international agierenden Terrorismus und des Anstiegs ihrer gewaltaffinen Sympathisanten in den vergangenen Jahren immer stärker auch zu einem prominenten Thema in der bundesdeutschen Sicherheits- und Integrationsdebatte geworden, das aber weitgehend separat von den oben beschriebenen Perspektiven behandelt wird (u. a. Ceylan/Kiefer 2013, 2017a; Kiefer et al. 2017; Toprak/Weitzel 2019).

Die Terroranschläge des 11. Septembers 2001 führten dazu, dass die in Gemeinden gelebte muslimische Religiosität zunehmend mit Misstrauen und als Integrationshindernis wahrgenommen wurde (vgl. Pollack/Müller 2013: 33 f., 38 ff., 56; Hafez/Schmidt 2015). Daraus resultierte die Verzahnung einer neu akzentuierten ,Islampolitik' mit einerseits repressiven sicherheits- und anderseits präventiven integrationspolitischen Maßnahmen als eine neue, über den deutschsprachigen Raum hinausgehende innergesellschaftliche Aufgabe (Tezcan 2016: 164). Diese Entwicklung wiederum führte zu einer verstärkten Untersuchung der Bedeutung von Religion für Integrations- und (zivil-)gesellschaftliche Beteiligungsprozesse, mit einem Fokus auf den Islam bzw. auf islamische Gemeinden und Verbände (u. a. Bertelsmann Stiftung 2008; Pollack/Müller 2013; Traunmüller 2014; Hafez/Schmidt 2015; El-Menouar 2017; Nagel/El-Menouar 2017). Seitens der Politik sind einige Studien in Auftrag gegeben worden, die sich zum Teil auf einzelne Bundesländer oder Berlin beziehen (Brettfeld/Wetzels 2007: 34 ff.; Haug et al. 2009; Stichs et al. 2010; Chbib 2011; Halm et al. 2012a bzw. Sauer/Halm 2013; Halm/Sauer 2015; für Berlin: Jonker/Kapphan 1999; Spielhaus/Färber 2006; Spielhaus/Mühe 2018). Die Untersuchungen kommen zu ähnlichen, nachfolgend skizzierten Ergebnissen.

Die zwei Studien Islamisches Gemeindeleben in Deutschland (Halm et al. 2012a) und Soziale Dienstleistungen der in der Deutschen Islam Konferenz vertretenen religiösen Dachverbände und ihrer Gemeinden (Halm/Sauer 2015) verdeutlichen, dass

\footnotetext{
${ }^{30}$ Siehe u. a.: Spuler-Stegemann (2002): Muslime in Deutschland; Küçükhüseyin (2002): Türkische politische Organisationen in Deutschland; Wunn (2007): Muslimische Gruppierungen in Deutschland. Ein Handbuch; Sökefeld (Hg.) (2008): Aleviten in Deutschland. Identitätsprozesse einer Religionsgemeinschaft in der Diaspora; Halm (2010): Muslimische Organisationen in Deutschland - Entwicklung zu einem europäischen Islam?; Meyer/Schubert (Hg.) (2011): Politik und Islam; Halm/Meyer (Hg.) (2013): Islam und die deutsche Gesellschaft; Rohe (2016): Der Islam in Deutschland. Eine Bestandsaufnahme; Hunger/Schröder (Hg.) (2016): Staat und Islam. Interdisziplinäre Perspektiven; Peucker (2016): Muslim Citizenship in Liberal Democracies. Civic and Political Participation in the West; Peucker/Ceylan (Hg.) (2017): Muslim Community Organizations in the West: History, Developments and Future Perspectives; Antes/Ceylan (Hg.) (2017): Muslime in Deutschland.
} 
die Arbeit und Angebote der rund 2.350 Moscheegemeinden in Deutschland weitestgehend von freiwillig Engagierten bzw. ehrenamtlich tätigen Personen getragen werden. Dirk Halm und Martina Sauer (2015) haben in ihrer Studie 38 Prozent der in der 18. Legislaturperiode in der Islamkonferenz vertretenen islamischen einschließlich alevitischen Dachverbände befragt und deren an den Sozialgesetzbüchern (SGB VIII, XI, XII) orientierten sozialen Dienstleistungen im Bereich der Kinder- und Jugend- sowie Altenhilfe - also keine religiösen Angebote - erhoben. Die Untersuchung zeigt, dass 94 Prozent der Gemeinden über Abteilungen für Kinder- und Jugendhilfe, 92 Prozent über einen Teilbereich für Frauen und 54 Prozent über Abteilungen für Senioren verfügen (Halm/Sauer 2015: 25). Dabei stehen einer Anzahl von 9.562 ehrenamtlich Tätigen 844 hauptamtliche Mitarbeiter gegenüber (ebd.: 36 ), wobei es sich bei letzteren häufig um das religiöse Personal handelt. Den Autoren zufolge kann das auf Kontinuität basierende Ehrenamt (ebd.: 41) fehlende hauptamtlich besorgte Arbeiten kompensieren, aber nicht komplett auffangen. Die große Anzahl von Gemeindenutzern begünstigt die Mobilisierung von Engagement. Eine „Proportionalität zwischen Gemeindegröße und Ehrenamt bezogen auf die abgefragten sozialen Dienstleistungen“ (ebd.: 40) ist jedoch nicht auszumachen.

Die Studie Islamisches Gemeindeleben in Deutschland (Halm et al. 2012a) kommt auf Basis einer Befragung der in Bezug auf Glaubensrichtungen, Herkunft und Größe sehr unterschiedlichen islamischen einschließlich alevitischen Verbände wie auch verbandsunabhängiger Organisationen zu dem Ergebnis, dass trotz der dominant ehrenamtlichen Strukturen eine starke Ausdifferenzierung der verbandlichen Organisationen und viele Gemeinsamkeiten festzustellen sind. Als zentrale Voraussetzung bzw. wesentlichen Einflussfaktor für die Handlungsfähigkeit und Bereitstellung von (nicht-)religiösen Angeboten der Verbände identifiziert die Studie die Existenz ,personeller, infrastruktureller und finanzieller Ressourcen“ (Halm et al. 2012a: 117). Dabei steht die Ressourcenausstattung (Räumlichkeiten, Immobilienbesitz, edukative und kulturelle Abteilungen) in einem proportionalen Verhältnis zur Verbandsgröße. Trotz dominant türkischer Prägung können die häufig seit über 20 Jahren bestehenden Verbände als herkunftsheterogen charakterisiert werden. Neben religiösen existieren zahlreiche nicht-religiöse Angebote wie Hausaufgabenhilfe, Sozial-, Erziehungs- und Gesundheitsberatung, wobei die Dienstleistungen nicht herkunftskulturdominiert oder in einem Konkurrenzverhältnis zueinander stehen (Halm et al. 2012a: 5-8, 116-119; Sauer/Halm 2013: 413-415). Grenzüberschreitende Aktivitäten sind eher selten und abhängig von der Ressourcenausstattung (Halm et al. 2012a: 7). Eine Integrationsresistenz lässt sich bei keinem der Verbände identifizieren: „Entsprechend der Theorie der multiplen Integration lassen sich bei den Gemeinden durchaus eine Hinwendung 
zur Aufnahmegesellschaft und assimilatorische Aktivitäten bei Beibehaltung herkunftskultureller Orientierungen und binnenorientierter Aktivitäten feststellen“ (Sauer/Halm 2013: 414). Die meisten der Verbände sind mit Akteuren der Aufnahmegesellschaft vernetzt, etwa durch die Involvierung im interreligiösen Dialog, wobei sich die Zusammenarbeit mit anderen Akteuren für verbandsunabhängige, schiitische und bosnische Gemeinden aufgrund deren geringerer Ressourcenausstattung und interner Aufgabendifferenzierung schwieriger gestalten könnte (Halm et al. 2012a: 119). Laut den Autoren der Studie kommt den Religionsbediensteten, in ihrer Funktion als Vertrauen genießende Multiplikatoren, ,eine Schlüsselrolle“ zu (ebd.: 5).

Auch die Ergebnisse von zwei durch die Stiftung Zentrum für Türkeistudien und Integrationsforschung (ZfTI) durchgeführten Studien (Halm/Sauer 2005; Sauer 2011) lassen insgesamt nicht auf Abschottungs- oder Segregationstendenzen der befragten Türkeistämmigen schließen. Eigenethnische Kontexte würden dann relevant, wenn der aufnahmegesellschaftliche Kontext - wie im Falle der Religion oder der migrationsspezifischen Problembearbeitung - keine Alternativen bereithalte (Sauer 2011: 223; Halm/Sauer 2005: 81). Hinsichtlich der Länderorientierung der Organisationen (Deutschland; Deutschland und Türkei; Türkei; internationale Ebene) ist eine Varianz nach Engagementbereichen und Organisationscharakter und eine zunehmende Bezugnahme auf Deutschland ersichtlich (Sauer 2011: 224). 55 Prozent der Engagierten charakterisieren ihre Organisationen als Zusammenschlüsse, die nicht auf die ethnische Herkunft oder Migrationssituation ausgerichtet $\operatorname{sind}^{31}$ (Sauer 2011: 221).

Die jüngste auf Basis eines detaillierten Gesprächsleitfadens durchgeführte Studie Islamisches Gemeindeleben in Berlin von Riem Spielhaus und Nina Mühe (2018) erfasst 60 Prozent der insgesamt 98 islamischen Vereine und Gebetsorte in Berlin, von denen 66 Prozent einem regionalen oder bundesweiten islamischen Dachverband angehören. Mit der Untersuchung wird die große Vielfalt der Aktivitäten der Gemeinden beleuchtet, die sich vornehmlich auf religiöse und soziale Dienstleistungen konzentrieren. Die Ermöglichung des Gemeinschaftsgebets und ritueller Praxis besitzen einen hohen Stellenwert. Neben (religiöser) Betreuungs- und Bildungsarbeit wie Nachhilfeunterricht und Sprachkurse für Deutsch und Arabisch (um religiöse Quellen besser verstehen zu können) für Kinder, Jugendliche und Erwachsene bieten über 80 Prozent der befragten Gemeinden besondere Freizeitaktivitäten

${ }^{31}$ Dabei variiert die Selbstbezeichnung der Organisationen in den einzelnen Engagementbereichen zwischen den Angaben 1.) eine ethnische, kulturelle oder religiöse Gemeinschaft $(37 \%), 2$.) eine herkunftsunabhängige Interessengemeinschaft $(\underline{35 \%}), 3$.) eine problemorientierte herkunftsunabhängige Selbsthilfeorganisation (20\%) oder 4.) eine migrationsbedingte Selbsthilfeorganisation zu sein (6\%) (Sauer 2011: 221; $\mathrm{n}=1.136$; Einfachnennung). 
für Jugendliche an. Dazu gehören z. B. Sportangebote wie Fuß- und Basketball (auch für junge Frauen), Computer-, Musik- und Folklorekurse, Museumsbesuche oder gesellige Zusammenkünfte. Weitere zielgruppenspezifische Angebote wie Diskussionsveranstaltungen und für auf den familiären, ehelichen und schulischen Kontext bezogene Beratungsangebote richten sich an Frauen, Ältere und Familien. Seit 2015 hat zudem die Arbeit mit Geflüchteten an Bedeutung gewonnen (Spielhaus/Mühe 2018: 44, 46, 50 ff.). Die Studie kommt zu dem Schluss, dass sich die Gemeinden in den letzten Jahren zunehmend geöffnet und ihre Vernetzung mit unterschiedlichen in Berlin ansässigen Akteuren aus der Politik, den Medien, anderen Vereinen und Religionsgemeinschaften forciert haben (ebd.: 110 ff.). Dabei beruhen ihre Aktivitäten größtenteils auf dem Einsatz von Ehrenamtlichen bzw. freiwillig Engagierten; der Wunsch nach mehr Professionalisierung durch Fördermittel wird insbesondere für die Jugendarbeit geäußert (ebd.: 55).

Die Ende 2020 veröffentlichte Studie Wohlfahrtspflegerische Leistungen von säkularen Migrantenorganisationen in Deutschland, unter Berücksichtigung der Leistungen für Geflüchtete (Halm et al. 2020) hat die Bedingungen analysiert, unter denen die Erbringung der sozialen Dienstleistungen in säkularen MSO erfolgt. Damit ist ein direkter Vergleich zu den bereits dargelegten Befunden der beiden Studien zu muslimischen und alevitischen Organisationen (Halm et al. 2012a; Halm/Sauer 2015) möglich. Die Untersuchung kommt zu dem Ergebnis, dass die säkularen MSO verschiedene migrationsspezifische Interessen vertreten und die Versorgung diverser Zielgruppen mit wohlfahrtspflegerischen Angeboten übernehmen (ebd.: 118). Im Vergleich zu den muslimischen und alevitischen Vereinigungen ist die Bandbreite der erbrachten Dienstleistungen unter den säkularen MSO jedoch deutlich größer, wobei dies auf die Verfügbarkeit von mehr hauptamtlichem Personal zurückzuführen ist. Obwohl die Zahl der gar nicht oder nur geringfügig mit Hauptamtlichen ausgestatten säkularen MSO überwiegt, lassen zahlreiche Organisationen auch einen „,beträchtlichen Professionalisierungsgrad“ (ebd.: 119) erkennen (vgl. auch Friedrichs et al. 2020: 45; I. 2.1). Während in den muslimischen und alevitischen Organisationen eher das Problem einer ausreichenden Qualifizierung der Ehrenamtlichen für deren adäquaten Einsatz und nicht deren Rekrutierung als solche eine wesentliche Schwierigkeit darstellt, wird für die säkularen MSO hingegen konstatiert, dass für diese in Hinblick auf die Erbringung sozialer Dienstleistungen die Gewinnung von Ehrenamtlichen eine zentrale Aufgabe darstellt (ebd.: 122), und zwar obwohl die säkularen Organisationen durchschnittlich über eine höhere Anzahl von Ehrenamtlichen als die religiösen verfügen. Die Autoren schlussfolgern, dass die religiösen MSO offenbar mehr Freiwillige rekrutieren können, was allerdings mit einem Qualifizierungsproblem verquickt ist, wohingegen die säkularen MSO „,eher einschlägig Interessierte und Qualifizierte gewinnen können. Hinzu kommt, 
dass die Herausforderungen der Fluchtmigration [...] den Bedarf an Freiwilligen insgesamt deutlich gesteigert haben können“ (ebd.).

Sowohl die Studien zu den religiösen als auch den säkularen MSO kommen zu dem Ergebnis, dass ressourcenstärkere und ausdifferenzierte Organisationen eher soziale Dienstleistungen anbieten als kleinere, und zwar unabhängig von einer spezifischen Finanzierungsart (ebd.: 121). Für eine Weiterentwicklung und langfristige Sicherstellung der Angebotserbringung wird die Finanzierung von dafür grundlegenden Organisationsstrukturen als notwendig erachtet (ebd.: 119).

\section{MSO im Spiegel von Engagementbeteiligung und „Sozialkapital“}

Im Folgenden sollen Ergebnisse von Studien zu nicht-religiösen und religiösen MSO fokussiert werden, die unter dem Aspekt der Engagementbeteiligung oder der Generierung von Sozialkapital zu betrachten sind. Unter dem Begriff des Sozialkapitals kann der Wert von sozialen Beziehungen hinsichtlich der sozialintegrativen Bedeutung für Einzelpersonen und den gesellschaftlichen Zusammenhalt, d. h. die Integrationsfähigkeit der Gesellschaft insgesamt beschrieben werden (II. 4.2.1). Robert Putnam (2000: 19) zufolge sind mit dem Begriff drei zentrale Dimensionen verknüpft: 1.) soziale Beziehungsstrukturen von Individuen und Gruppen, 2.) partikulares und generalisiertes Vertrauen und 3.) Normen der Reziprozität ${ }^{32}$. Die drei Größen sind interdependent und tragen zusammen zur Bildung von Sozialkapital bei. In einer sich ethnisch und religiös pluralisierenden Gesellschaft werden die positiven Effekten von Sozialkapital wie kooperatives, unterstützendes, vertrauensbasiertes und institutionell effektives Handeln besonders wertgeschätzt. In der Sozialkapital-Debatte hochrelevant ist die spezifische Art von in Vereinigungen oder Gemeinschaften vorzufindenden Beziehungen, bei denen zwischen einem innerhalb einer Gruppe bestehenden bindenden (bonding) und einem überbrückenden (bridging), d. h. sich auf andere soziale Gruppen beziehenden Sozialkapital unterschieden wird (II. 4.2.3). In diesem Kontext ist Sozialkapital zu einem Schlüsselkonzept für die Beurteilung der Sozialintegrationswirkung auch von MSO avanciert. $^{33}$

32 ,[S]ocial capital refers to connections among individuals - social networks and the norms of reciprocity and trustworthiness that arise from them" (Putnam 2000: 19).

${ }^{33}$ Allerdings ist darauf hinzuweisen, dass das Ausmaß von in Vergemeinschaftungen vorzufindender Engagementbeteiligung nicht automatisch mit der Ausbildung von Sozialkapital und Netzwerkstrukturen im Sinne Putnams gleichzusetzen ist (Halm 2015: 67). Diese Schlussfolgerung scheint fälschlicherweise häufig aufgrund der hohen Attraktivität des gesellschaftspolitisch verheißungsvollen Catch-all-Begriffs ,Sozialkapital“ verbreitet zu sein. 
In den letzten Jahren ist ein Boom von Analysen der quantitativen Sozialforschung festzustellen, die auf Basis großer Datensätze die Bedeutung unterschiedlicher religiöser Traditionen für die Einbindung von Individuen in (zivil-)gesellschaftliche Beziehungsstrukturen sowie die Ausbildung von Vertrauen auf internationaler Ebene untersuchen (Traunmüller 2008, 2011, 2012, 2014, 2018; Roßteutscher 2009, 2011; Pickel/Gladkirch 2011; Pollack/Müller 2013; Pickel 2014; Liedhegener 2016; Liedhegener et al. 2019). Für Deutschland stellt sich die Frage nach Effekten von Religion auf die Einbindung von Individuen in soziale Netzwerke und Zusammenschlüsse und die Konstituierung von Vertrauen insbesondere im Zusammenhang mit muslimischer Religiosität (Heinrich-Böll-Stiftung et al. 2007; Haug et al. 2009: 163-172, 253-282; Pollack/Müller 2013: 46-56; El-Menouar 2013; Vopel/El-Menouar 2015; El-Menouar 2017; Nagel/El-Menouar 2017).

Im Gegensatz dazu stellen quantitative empirische Studien auf der Mesoebene, die dezidiert und detailorientiert die Produktion von (religiösem) Sozialkapital insbesondere in Bezug auf die Bonding- und Bridging-Ausprägungen in religiösen und ethnischen migrantischen Vereinigungen in Deutschland untersuchen, ein Desiderat dar. Zwar thematisieren die oben erwähnten Studien zu den Organisationsstrukturen sowie Angeboten von islamischen Organisationen und Dachverbänden (Spielhaus/Färber 2006; Halm et al. 2012a; Halm/Sauer 2015; Spielhaus/Mühe 2018 etc.) die integrativen Beiträge und die herkunftslandbezogenen und aufnahmegesellschaftlichen Ausrichtungen; sie sind aber nicht dezidiert dem Blickwinkel von Bridging- und Bonding-Sozialkapital zuzurechnen, wenngleich bei dem Versuch der Einordnung der Studien deutlich wird, dass die Untersuchungsfoki in ihren Erkenntnisansprüchen nicht trennscharf voneinander abzugrenzen sind und die Studien daher in einer thematischen Weitwinkelperspektive auch unter dem Aspekt von bonding und bridging betrachtet werden könnten.

Detailliertere Analysen für die Mesoebene liegen für unterschiedliche religiöse Minderheiten in Form von qualitativen Fallstudien vor, die die Leistungsfähigkeit religiöser MSO hinsichtlich gesamtgesellschaftlich relevanter integrativer Effekte unterschiedlich einstufen. So lässt sich resümieren, dass binnenorientierte Unterstützungsangebote und Bedürfnisorientierungen an den eigenen Mitgliedern nicht immer mit nach außen gerichteten Aktivitäten und Kooperationen sowie dem Einbezug anderer Gruppen einhergehen (Nagel 2013a, 2015a, 2016; Elwert 2015a; Arens et al. 2016; Baumann 2016, 2018).

Entsprechend fallen Studienergebnisse zu Wechselwirkungen zwischen Zivilgesellschaft und Religion uneindeutig, teilweise auch widersprüchlich aus. Sie verdeutlichen die Abhängigkeit der Entwicklung religiösen Sozialkapitals von je spezifischen Verhältnissen zwischen politischem System, Zivilgesellschaft und 
Religion (Liedhegener/Werkner 2011a, b; Pollack 2002; Freitag 2006; Roßteutscher 2009; Traunmüller 2008, 2012; Von Beyme 2015; Arens et al. 2016; s. dazu auch II. 3.1 und II. 3.2). Die Verflochtenheit von Zivilgesellschaft und Religion ist mithin nicht nur theoretisch, sondern folgerichtig auch empirisch ambivalent; die sozialkapitalrelevanten Effekte von Religion zeichnen sich durch eine ,fundamentale Kontextabhängigkeit“ (Traunmüller 2012: 22) aus. Religiös eingebettetes Engagement ist daher hinsichtlich seiner zivilgesellschaftlichen Qualität und der Stärkung des gesellschaftlichen Zusammenhalts differenziert zu erforschen, Beziehungen zwischen Religion und Sozialkapital lassen sich nicht eindeutig als Wirkungsoder Kausalrichtungen bestimmen. Festzuhalten bleibt, dass sich Sozialkapital bei einigen religiösen Gemeinschaften eher gruppenintern integrierend (,bonding“) und bei anderen eher als zwischen unterschiedlichen gesellschaftlichen Gruppen brückenbildend (,,bridging“) ausprägen wird. ${ }^{34}$

Im Folgenden werden für die vorliegende Arbeit relevante Befunde zur Engagementbeteiligung von Angehörigen unterschiedlicher religiöser Traditionen mit einer Konzentration auf den Islam und der Flüchtlingshilfe dargelegt. Zudem erfolgt eine Zusammenfassung der zentralen Ergebnisse von dezidiert unter dem Aspekt des Sozialkapitals verhandelten qualitativen Studien zu Integrationsleistungen und politischer Beteiligung von Angehörigen verschiedener ethnischer Gemeinschaften in Deutschland. Im Anschluss daran werden Ergebnisse qualitativer religionswissenschaftlicher Fallbeispiele zu Aktivitäten und zum Vernetzungsverhalten verschiedener religiöser Gemeinschaften in der Schweiz und in Deutschland dargelegt.

\section{Engagementbeteiligung von Muslimen}

Studien zu Muslimen und deren Engagementbeteiligung sowie Ausbildung von Sozialkapital in organisierten Kontexten in Deutschland sind überschaubar. Dem Freiwilligensurvey 2014 zufolge verfügen islamische Religionsgemeinschaften mit 27 Prozent über einen deutlich geringeren Anteil an freiwillig Engagierten als die christlichen Religionsgemeinschaften (Katholische Kirche: $49 \%$; Evangelische Kirchen: $49 \%$ ). Zurückgeführt wird dieses Ergebnis auf die höheren Mitgliederquoten der großen christlichen Kirchen, auf die für sie entsprechend vorteilhafteren Mobilisierungsbedingungen, ihre starken zivilgesellschaftlichen (Macht-)Positionen und wirtschaftliche Stärke. Muslimische Vereinigungen seien hingegen mit öffentlichen, staatlichen Akteuren und zivilgesellschaftlichen Organisationen weniger vernetzt (Vogel/Hameister 2016: 239, 246). Diese Befunde korrespondieren mit Ergebnissen anderer Studien zum Zusammenhang von Religion und Engagement, in

\footnotetext{
${ }^{34}$ Siehe zu diesen Ausführungen auch Klie 2018: 449 f.
} 
denen bei Kontrolle sozioökonomischer Faktoren ebenfalls eine deutlich geringere, unterdurchschnittliche Ausprägung von Engagement und zivilgesellschaftlicher Einbindung von Muslimen in Deutschland und anderen europäischen Ländern berichtet wird (Traunmüller 2008, 2012, 2014, 2018; Haug et al. 2009; Pollack/Müller 2013; Liedhegener et al. 2019; für die Schweiz: Stadelmann-Steffen et al. 2010; Liedhegener et al. 2019).

Im Kontrast zu den oben dargelegten Befunden stehen die Ergebnisse des Religionsmonitors 2017, die zeigen, dass sich Muslime mit einem Anteil von 44 Prozent in Deutschland deutlich stärker in der Flüchtlingshilfe freiwillig engagieren als dies Christen (21\%) und Konfessionslose tun (17\%). Es kann nachgewiesen werden, dass die Zugehörigkeit zum Islam einen signifikanten positiven Effekt auf das Engagementverhalten der Muslime hat (Nagel/El-Menouar 2017: 40 ff.). Die Befragungsergebnisse weisen sowohl für Christen als auch Muslime eine starke Korrelation zwischen Gemeindebindung und freiwilliger Hilfeleistung für Geflüchtete nach. Dabei ist das regelmäßige informelle, soziale Eingebundensein im Gemeindekontext außerhalb religiöser Zeremonien, gemessen über die Häufigkeit des Gemeindebesuchs, und nicht der regelmäßige Besuch von Sonntagsgottesdiensten oder Freitagspredigten für ein höheres Engagementniveau ausschlaggebend. ${ }^{35}$ Diese Gemeindeanbindung sorgt bei der betreffenden Gruppe der Muslime für einen Engagiertenanteil von 72 Prozent, bei der Gruppe der Christen von 40 Prozent. Das Fehlen der Gemeindeanbindung bewirkt ein Absinken der Prozentzahlen auf 40 Prozent bei den Muslimen und auf 17 Prozent bei den Christen (ebd.: 44). In Bezug auf Engagementbeteiligung zeigt sich ein schwacher, aber nicht eindeutig linearer Zusammenhang zwischen der Häufigkeit der Teilnahme am Freitagsgebet bzw. am Gottesdienst. Befragte Christen, die selten bis nie Gottesdienste besuchen, engagieren sich seltener in der Flüchtlingshilfe. Unter ihnen ist der Anteil der Flüchtlingshelfer mit 39 Prozent unter denjenigen Personen am höchsten, die ein- bis dreimal im Monat in die Kirche gehen; der Anteil der Engagierten geht bei einer regelmäßigen Gottesdienstteilnahme (jeden Sonntag) auf 33 Prozent zurück. Bei den Muslimen zeigt sich ein etwas deutlicherer Zusammenhang: Regelmäßige Freitagsgebetsbesucher (jeden Freitag) werden mit einem Anteil von 56 Prozent überdurchschnittlich häufig in der Flüchtlingshilfe aktiv; interessanterweise fällt unter Muslimen, die nie in eine Moschee gehen, der Anteil Engagierter mit 41 Prozent ebenfalls relativ hoch aus.

\footnotetext{
${ }^{35}$ Auch Liedhegener et al. kommen in ihrer KONID-Studie (,KONID“ = „Konfigurationen individueller und kollektiver religiöser Identitäten und ihre zivilgesellschaftlichen Potentiale“) zu dem Ergebnis: „Vor allem die Einbindung in Religion und die individuelle Religiosität beim Einzelnen besitzen eine förderliche Wirkung auf das zivilgesellschaftliche Engagement und damit die Lebendigkeit der Zivilgesellschaft“ (Liedhegener et al. 2019: 29).
} 
Hier zeigt sich der nicht eindeutige, ambivalente Charakter des für die Erklärung von Engagementbeteiligung klassischerweise herangezogenen Prädiktors „Gottesdienstbesuch“ in den betreffenden religiösen Gemeinden. Der signifikante Effekt der alltäglichen sozialen und informellen Gemeindeanbindung auf die Engagementbereitschaft macht deutlich, dass die Gemeinden als soziale Orte und Engagementplattformen fungieren (ebd.: 29 f.). Zugleich ist festzuhalten, dass die religiösen Gemeinden nicht das alleinige Vehikel für Engagementbeteiligung von Christen und Muslimen darstellen: So werden von 40 Prozent der befragten Muslime, die nie am Freitagsgebet teilnehmen und nicht in Moscheen verkehren, Engagementmöglichkeiten und -angebote auch außerhalb der Gemeinden genutzt (ebd.: 30).

Insgesamt verweist der Forschungsstand auf eine Diskrepanz zwischen dem repräsentativen Befund einer deutlich geringeren Engagementbeteiligung von Muslimen (im Vergleich zu Autochthonen) auf der Individualebene gegenüber dem festgestellten Sachverhalt, dass muslimisches Engagement eine tragende Säule bei der Bewältigung der alltäglichen Arbeiten in den islamischen Gemeinden darstellt (Halm et al. 2012a: 421; Halm/Sauer 2015; Spielhaus/Mühe 2018).

\section{Sozialkapital und Integration durch ethnische und religiöse Gemeinschaften: Qualitative Fallstudien}

\section{Ethnische Vereinigungen}

Qualitative Analysen von verschiedenen auf ethnischer Zugehörigkeit basierenden Vereinigungen beziehen sich auf das dort vorhandene zivilgesellschaftliche Engagement, die Produktion von Sozialkapital und das politische Partizipationsverhalten. Dies impliziert die populäre Frage nach der Brückenfunktion der Organisationen in Hinblick auf mehr soziale und politische Teilhabe der Mitglieder.

Einzelne Fallstudien legen dar, wie Eingliederungsprozesse und Teilhabe von in Vereinen organisierten Einwanderergruppen je nach Ausmaß und Qualität der Netzwerke, der Interaktionen mit dem deutschen Umfeld sowie Milieuzugehörigkeiten disparat ausfallen (Thränhardt/Hunger 2000). Im Performanz-Vergleich wird auf viele Erfolgsgeschichten verwiesen, die mit einem beträchtlichen gesellschaftspolitischen Mitgestaltungs- und Integrationswillen und der Entstehung von brückenbildendem Sozialkapital, das Wege in die Aufnahmegesellschaft ebnet, erklärt werden. Dadurch seien auch Erfolge in Bildung, Beruf, politische und soziale Partizipation sowie Selbstbestimmung des eigenen Lebens realisierbar (Thränhardt 2000a: $11 \mathrm{ff}$.). Die Untersuchung spezifischer Eigenschaften von türkisch-alevitischen, vietnamesischen, spanischen und italienischen Selbsthilfevereinigungen - in Hinblick auf deren potenziell mobilisierenden, akkulturierenden oder exkludierenden 
Funktionen - verdeutlicht, dass Wenn-Dann-Schlussfolgerungen nicht möglich sind (Thränhardt 2000b). Stattdessen zeigt eine synoptische Bilanz der im Sammelband von Karin Weiss und Dietrich Thränhardt (2005) versammelten Analyseergebnisse, dass eine differenzierte Betrachtung der sehr unterschiedlich ausfallenden Selbstorganisations- bzw. Selbsthilfestrukturen notwendig ist. Diese werden v. a. geprägt durch die jeweiligen Ziele, durch Organisations- und Professionalisierungsgrade, durch ethnische und soziale Zusammensetzungen sowie durch die Beziehungen zu deutschen Organisationen. Als diese wiederum beeinflussende Größen werden die Bedingungen der ethnischen Gruppe in Deutschland, nationale und regionale Rahmenfaktoren sowie Beziehungen zum Herkunftsland identifiziert (Tab. 2.4; Weiss/Thränhardt 2005b: 34-37; s. zu den nationalen und regionalen Rahmenbedingungen auch II. 3.2).

Tab. 2.4 Einflussgrößen für die Ausgestaltung von Selbstorganisations- bzw. Selbsthilfestrukturen

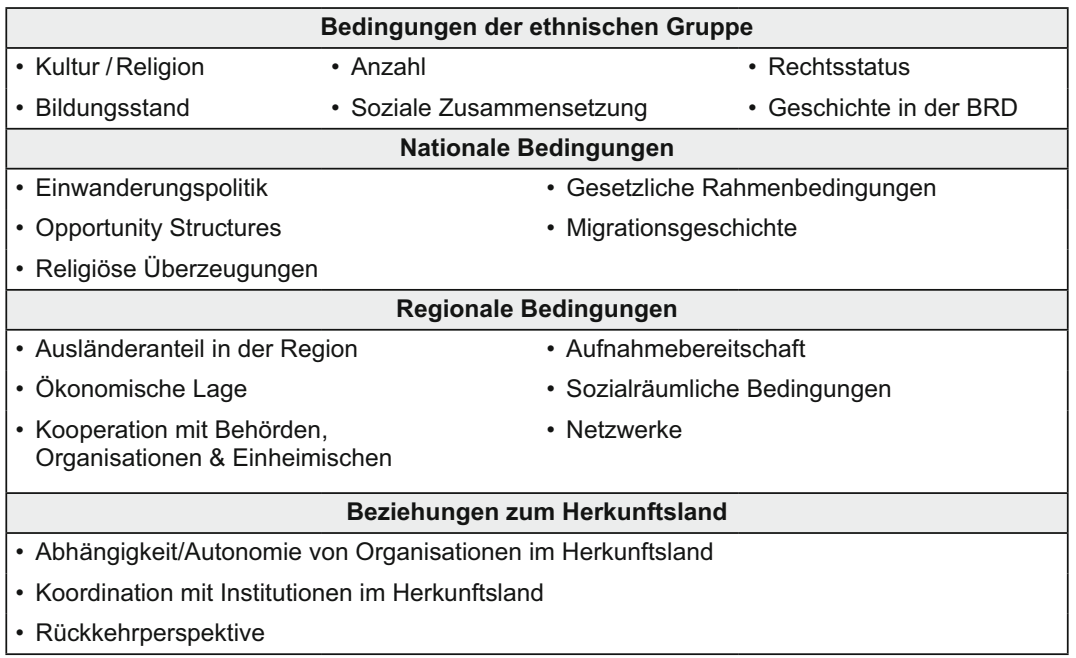

Quelle: Nach Weiss/Thränhardt 2005b: 37.

Auch anderer Untersuchungen zu ethnischem oder migrationsspezifischem Sozialkapital (Rauer 2004; Diehl 2004; Berger et al. 2004) zeigen, dass Ergebnisse bei unterschiedlichen ethnischen Gruppen divergieren und z. T. auch uneindeutig ausfallen. Entsprechend verbietet es sich, pauschale Aussagen zu Bridging- und 
Bonding-Ausrichtungen und damit einhergehenden oder ausbleibenden integrativen Effekten von MSO hinsichtlich der politischen und sozialen Beteiligung der in ihnen involvierten Individuen zu treffen. Vielmehr prägen sich Funktionen und Wirkungen von MSO fallspezifisch und kontextabhängig aus.

\section{Religiöse Migrantengemeinden}

Martin Baumann (2016) untersucht anhand von in der Schweiz durchgeführten qualitativen Fallbeispielen auf Basis einer kritischen Auseinandersetzung mit der idealtypischen Differenzierung zwischen ,passiver“ und ,aktiver“ Integration, die Wolfgang Vortkamp (2008) vorgeschlagen hat (vgl. Baumann 2016: 78 ff., 108 ff.), die (des-)integrativen Potenziale religiöser Immigrantenvereine, und zwar am Beispiel von Hindu-Tempeln, Moscheen und christlich-orthodoxen Kirchen. Dabei bezieht er auch die Mikroebene von religiösen Funktionsträgern - Imamen, Priestern und Vorstandspersonen - sowie Prozesse, die die Errichtung religiöser Sakralbauten betreffen, mit ein. Baumann kommt zu dem Schluss, dass die Aktivitäten und Dienstleistungen der Gemeinden überwiegend nach innen gerichtet sind und damit die Form der „Binnenintegration“ und des Bonding-Sozialkapitals dominiert, was wiederum eine wichtige Voraussetzung für eine gesamtgesellschaftliche Teilhabe darstelle: ,[G]erade diese Unterstützung und Stärkung in der eigenen Gemeinschaft und Gruppe kann wichtige Vorbedingung sein, um ein über das Nahfeld hinausgehendes soziales oder zivilbürgerliches Engagement zu entwickeln. Gruppenbezogenes bonding-Sozialkapital kann zu gruppenübergreifenden bridging-Sozialkapital anleiten“ (Baumann 2016: 111; Herv. i. Orig.). Die Funktionsträger besäßen aufgrund ihrer hervorgehobenen und anspruchsvollen Rolle als Berater, Repräsentanten der Gemeinschaft und Kontaktpersonen für Politik, Medien und andere religiösen Gemeinden erhöhte gesellschaftliche Teilhabechancen und Mitspracherechte (ebd.: 90, 111 f.; vgl. auch Halm et al. 2012a: 5). Dabei fielen Offenheit nach außen, Kommunikationsbedürfnisse mit der Umwelt, gesellschaftspolitische Mitgestaltungs- und Partizipationsansprüche und Verantwortungsübernahmen in moscheegebundenen religiösen Jugend- und Reformgruppen deutlich anders aus als in konservativen Hindu-Tempeln, die sich stark auf traditionelle Werte, Macht- und Kastengefüge bezögen (ebd.: 94 ff.).

Die Identifizierung der Aktivitäten der religiösen Immigrantenvereine als binnenorientierte, auf die Zuwanderungscommunity gerichtete Tätigkeiten korrespondiert mit jüngeren, im deutschen Kontext im Rahmen des Forschungsprojekts Religion vernetzt (2009-2014) in Nordrhein-Westfalen zu religiösen Migrantengemeinden durchgeführten qualitativen Fallstudien (Nagel 2013a, 2015a; Elwert 2015a). Im Rahmen dieses Projekts sind sowohl das Vernetzungsverhalten von 
religiösen Migrantengemeinden nach außen als auch die binnenorientierten Unterstützungsangebote unter einer potenzial- und beziehungsorientierten Perspektive analysiert worden. In den Blick gerieten damit soziale Dienstleistungen für die eigenen Mitglieder (und darüber hinausgehende Personengruppen) sowie Interaktionen mit anderen religiösen und nicht-religiösen Akteuren der Aufnahmegesellschaft. Die Untersuchungen erfolgten entlang einer systematischen Unterscheidung von relevanten internen und externen, strukturellen und ideellen Einflussfaktoren, die auch für den empirischen Teil der vorliegenden Arbeit bedeutsam sind (III. 6.1). Interne Faktoren betreffen das theologische Selbstverständnis, die Migrationsgeschichte, die Sozialstruktur und den demografischen Wandel. Externe Faktoren beziehen sich auf rechtliche Rahmenbedingungen, Anreize und Gelegenheitsstrukturen, das organisationale Feld und öffentliche Diskurse (Tab. 2.5). Mit dem Untersuchungsdesign sollten „kausale Mechanismen“ (Nagel/Plessentin 2015: 261), die das zivilgesellschaftliche Verhalten befördern oder hemmen, aufgedeckt werden.

Anhand eines netzwerkanalytischen Modells erfolgte ein Vergleich von einzelnen Fallstudien zu ethnoreligiösen Gruppen in Nordrhein-Westfalen - tamilischen Hindus, thailändischen Buddhisten, koreanischen Freikirchen, russlanddeutschen Mennoniten, syrisch-orthodoxen Christen, Eziden ${ }^{36}$ - sowie Moscheegemeinden, Neo-Muslimen und interreligiösen Initiativen. In der Summe wurde herausgefunden, dass religiöse Migrantengemeinden mit einer großen Bandbreite von Angeboten aufwarten, die eine ausgeprägte Binnenorientierung bzw. Bedürfnisorientierung an den eigenen Mitgliedern erkennen lassen. So sind neben den rituellen Aufgaben drei typische Angebotsschwerpunkte festzustellen: 1.) die Pflege der Herkunftssprache und -kultur, 2.) der Ausgleich sozioökonomischer Nachteile der Gemeindemitglieder, sowie 3.) die kollektive Bewältigung traumatischer Erfahrungen von Ausgrenzung und Vertreibung in den Herkunftsländern. Dabei sei das zielgruppenorientierte Angebotsspektrum nicht mit Abschottungstendenzen zu verwechseln, sondern gleiche dem von etablierten kirchlichen Ortsgemeinden,

\footnotetext{
${ }^{36}$ Siehe zu den zivilgesellschaftlichen Potenzialen der einzelnen religiösen Migrantengemeinden die Beiträge von Sandhya Marla(-Küsters) (2013, 2015) zu den tamilischen Hindu-Tempeln; von Ann-Kathrin Wolf (2015) zu den thailändisch-buddhistischen Zentren; von Sabrina Weiß $(2013,2015)$ zu den koreanischen Freikirchen; von Frederik Elwert (2013, 2015a, b) zu den mennonitischen Gemeinden russlanddeutscher Aussiedler; von Ulf Plessentin (2015) zur Syrisch-Orthodoxen Kirche; von Thorsten Wettich (2015) zur ezidischen Community; von Piotr Suder (2013, 2015) zu Moscheevereinen; von Karin (Mykytjuk-)Hitz (2013, 2015) zu neomuslimischen Akteuren sowie von Nelly C. Schubert $(2013,2015)$ zu interreligiösen (Dialog-)Initiativen.
} 
Tab. 2.5 Einflussfaktoren für Angebotsausrichtungen und Vernetzungsverhalten von religiösen Migrantengemeinden in NRW

\begin{tabular}{|c|c|c|}
\hline $\begin{array}{l}\text { Interne Einfluss- } \\
\text { faktoren (Innere } \\
\text { Verfasstheit } \\
\text { der Religions- } \\
\text { gemeinschaft) }\end{array}$ & Erläuterung & $\begin{array}{l}\text { Ausdifferenzierungen/ } \\
\text { Ergebnisse der Fallstudien }\end{array}$ \\
\hline $\begin{array}{l}\text { Theologisches } \\
\text { Selbstverständ- } \\
\text { nis (intern/ideell) }\end{array}$ & $\begin{array}{l}\text { - Ekklesiologische Vorstellun- } \\
\text { gen: theologische Konstruk- } \\
\text { tion sozialer Unterschiede nach } \\
\text { innen (z. B. Kasten; Spezialisten } \\
\text { vs. Laien) } \\
\text { - Umgang mit (nicht-)religiösen } \\
\text { Anderen } \\
\text { - Religiöser Unterstützungsethos: } \\
\text { Heilserlangung durch Hilfe- } \\
\text { leistungen für Andere }\end{array}$ & $\begin{array}{l}\text { 1. Ausprägung einer religiösen Solidar- } \\
\text { ethik } \\
\text { 2. Von theologischem, historisch } \\
\text { geprägtem Selbstverständnis } \\
\text { abgeleiteter Umgang mit anderen } \\
\text { (religiösen) Gruppen } \\
\text { (symbolische Grenzziehungen; } \\
\text { Verweis auf theologische Differen- } \\
\text { zen; Skepsis ggü. Zusammenarbeit } \\
\text { mit Amtskirchen, staatl. Institutionen } \\
\text { etc.) } \\
\text { 3. Theologische Innovationen } \\
\text { (Laisierung: Selbstermächtigung } \\
\text { religiöser Laien hinsichtlich der } \\
\text { Schriftenauslegung und Ritual- } \\
\text { ausübung; } \\
\text { Theologisierung: systematische } \\
\text { Reflexion und Kanonisierung } \\
\text { tradierter religiöser Vorstellungen } \\
\text { und Gebräuche) }\end{array}$ \\
\hline $\begin{array}{l}\text { Migrations- } \\
\text { geschichte } \\
\text { (intern/ideell; } \\
\text { strukturell) }\end{array}$ & $\begin{array}{l}\text { Religiöse Selbstorganisation im } \\
\text { Aufnahmeland durch Erfahrung } \\
\text { von Ausgrenzung und Vertrei- } \\
\text { bung als religiöse Minderheit in } \\
\text { den Herkunftsländern } \\
\text { - Erloschene Rückkehrhoffnungen }\end{array}$ & $\begin{array}{l}\text { 1. Status der Religionsgemeinschaft } \\
\text { im Herkunftsgebiet } \\
\text { 2. Zentrale Auswanderungsgründe } \\
\text { 3. Aussicht auf Rückkehr }\end{array}$ \\
\hline $\begin{array}{l}\text { Sozialstruktur } \\
\text { (intern/strukturell) }\end{array}$ & $\begin{array}{l}\text { - Bildungs- und Erwerbsstatus } \\
\text { der Gemeindemitglieder bestim- } \\
\text { men sozioökonomischen Hand- } \\
\text { lungsspielraum und Fähigkeit zur } \\
\text { Selbstorganisation ( } \rightarrow \text { schlechte } \\
\text { finanzielle Ausstattung der } \\
\text { meisten religiösen Migranten- } \\
\text { gemeinden) } \\
\text { - Mangelnde Sprachkenntnisse } \\
\text { - Habituelle Zurückhaltung der } \\
\text { ersten Generation } \\
\text { - Bedürfnislagen (z. B. Bildungs- } \\
\text { förderung, Sozialberatung, } \\
\text { Arbeitsvermittlung) }\end{array}$ & \\
\hline $\begin{array}{l}\text { Demografischer } \\
\text { Wandel } \\
\text { (intern/strukturell) }\end{array}$ & $\begin{array}{l}\text { Unterschiedliche Ressourcen- } \\
\text { ausstattung und Bedürfnislagen } \\
\text { der 1. und 2. Generation } \rightarrow \\
\text { Generationskonflikte } \\
\text { (2. Gen. = ökonomische } \\
\text { Aufwärtsmobilität, Aufnahme- } \\
\text { landorientierung, sehr gute } \\
\text { Sprachkenntnisse, erweiterte } \\
\text { Teilhabeansprüche) }\end{array}$ & $\begin{array}{l}\text { 1. Generationenwechsel (klare Unter- } \\
\text { scheidung von Generationenkohor- } \\
\text { ten als eigenständige Milieus mit } \\
\text { verschiedenen Bedürfnislagen) } \\
\text { 2. Siedlungsstruktur (großes Einzugs- } \\
\text { gebiet der Religionsgemeinschaften } \\
\text { vs. starke räumliche Konzentration) } \\
\text { 3. Heiratsverhalten }\end{array}$ \\
\hline
\end{tabular}


Tab. 2.5 (Fortsetzung)

\begin{tabular}{|c|c|c|}
\hline $\begin{array}{l}\text { Externe } \\
\text { Einflussfaktoren } \\
\text { (Bedingungen } \\
\text { und Einflüsse aus } \\
\text { der Umwelt) } \\
\end{array}$ & Erläuterung & $\begin{array}{l}\text { Ausdifferenzierungen/ } \\
\text { Ergebnisse der Fallstudien }\end{array}$ \\
\hline $\begin{array}{l}\text { Rechtliche } \\
\text { Rahmenbedin- } \\
\text { gungen } \\
\text { (extern/strukturell) }\end{array}$ & $\begin{array}{l}\text { Organisation als Körperschaft des } \\
\text { öffentlichen Rechts (KdöR) oder } \\
\text { eingetragener Verein (e.V.) } \\
(\rightarrow \text { Erhöhung der Selbst- } \\
\text { organisationskompetenz durch } \\
\text { Mitgliedsbeiträge, Weisungsstruk- } \\
\text { turen; Religionsunterricht an staat- } \\
\text { lichen Schulen) }\end{array}$ & \\
\hline $\begin{array}{l}\text { Anreize und } \\
\text { Gelegenheits- } \\
\text { strukturen } \\
\text { (extern/strukturell) }\end{array}$ & $\begin{array}{l}\text { - Finanzielle Förderung (staatliche } \\
\text { Zuschüsse für freie Wohlfahrts- } \\
\text { pflege; Ausschreibungen von } \\
\text { Stiftungen) } \\
\text { - Politische Gelegenheitsstruktu- } \\
\text { ren (Integrationsräte als Forum } \\
\text { politischer Mitwirkung; Integra- } \\
\text { tionsbüros als administrative } \\
\text { Ansprechpartner) }\end{array}$ & \\
\hline $\begin{array}{l}\text { Organisatio- } \\
\text { nales Feld } \\
\text { (extern/strukturell) }\end{array}$ & $\begin{array}{l}\text { - Religiöse Organisation der } \\
\text { Umwelt: materielle/personelle } \\
\text { Unterstützung durch Kirchen, } \\
\text { Wohlfahrtsverbände }(\rightarrow \text { plura- } \\
\text { les religiöses Feld ist historisch } \\
\text { durch Strukturen der etablierten } \\
\text { Kirchen vorgeprägt) } \\
\text { - Transnationale Ausprägung: } \\
\text { Austausch mit religiösen Organi- } \\
\text { sationen im Herkunftsland }\end{array}$ & $\begin{array}{l}\text { 1. Zentralisierungsprozesse (Formie- } \\
\text { rung nationaler Dachverbände, } \\
\text { Schaffung von Bistümern; Zusam- } \\
\text { menschluss der Spitzen von Dach- } \\
\text { verbänden bzw. kirchlicher Leitungs- } \\
\text { ebenen) } \\
\text { 2. Transnationale Konstellation (grenz- } \\
\text { überschreitende Beziehungen; } \\
\text { Austausch mit anderen Diaspora- } \\
\text { Standorten; ausgeprägte Diaspora- } \\
\text { Orientierung) }\end{array}$ \\
\hline $\begin{array}{l}\text { Öffentliche } \\
\text { Diskurse } \\
\text { (extern/ideell) }\end{array}$ & $\begin{array}{l}\text { Beobachtungen durch und Miss- } \\
\text { trauen der Aufnahmegesellschaft: } \\
\text { - Gefährdungsdiskurs } \\
\text { - Affinitätsdiskurs (prinzipielle } \\
\text { Ähnlichkeit und Übereinstim- } \\
\text { mung mit Werten der Aufnahme- } \\
\text { gesellschaft) } \\
\text { - Exotisierungsdiskurs (pauschal } \\
\text { positive Wahrnehmung; wohl- } \\
\text { wollendes Interesse) }\end{array}$ & $\begin{array}{l}\text { Ausrichtung der Diskurse bestimmt } \\
\text { Schwerpunktsetzung in Außendarstel- } \\
\text { lungen der Religionsgemeinschaften } \\
\text { (z. B. Gefährdungsdiskurs: Akzentuie- } \\
\text { rung von Umfang und Nützlichkeit der } \\
\text { Angebote von Moscheegemeinden; } \\
\text { Beziehungspflege im inter- und außer- } \\
\text { religiösen Kontext) }\end{array}$ \\
\hline
\end{tabular}

Quelle: Eigene Zusammenstellung nach Nagel 2015b: 27-31; Nagel/Plessentin 2015: 246-261; Nagel 2016. 
indem es auf religiösen Weltbildern und daraus abgeleiteten solidarethischen Normen und zudem auf einer geteilten Migrationsgeschichte und sozialer Kontrolle basiere (Nagel/Plessentin 2015: 244). ${ }^{37}$ Hinsichtlich des Vernetzungsverhaltens der Gemeinden sind im Vergleich der Fallstudien hingegen deutliche Unterschiede identifiziert worden, die auf unterschiedliche, historisch geprägte Kirchenverständnisse zurückzuführen seien. Zudem ließen die nichtmuslimischen Gruppen alle eine mehr oder weniger stark ausgeprägte Abgrenzung gegenüber dem Islam erkennen (ebd.: 250).

In der Summe kommen die fallbezogenen Untersuchungen zu dem Ergebnis, dass bei der Analyse der Konstellationen keine eindeutigen Kausal- und Wirkungsrichtungen zwischen Einflussfaktoren (Ursache) und den Angeboten und Vernetzungen (Wirkungen), sondern vielmehr „Ursachenbündel [festzustellen] sind, die zur Herausbildung bestimmter Angebote und Vernetzungen führen“ (ebd.: 262). Insbesondere interne Eigendynamiken und Entwicklungsprozesse religiöser Migrantengemeinden, die sich externen Einflussnahmen teilweise gänzlich entzögen, beeinflussten deren zivilgesellschaftlichen Potenziale maßgeblich. Multikausale Erklärungen und das komplexe Zusammenwirken unterschiedlicher Einflussfaktoren bestimmen somit das Angebots- und Beziehungsspektrum religiöser Migrantengemeinden: „Die zentrale Stellschraube zur Aktivierung der zivilgesellschaftlichen Potentiale religiöser Migrantengemeinden gibt es nicht“" (ebd.).

Als Fazit ist abschließend festzuhalten, dass aufgrund der Verschiedenheit der jeweils untersuchten ethnischen und religiösen Gemeinschaften und unterschiedlicher Erhebungsdesigns eine Vergleichbarkeit der Studienergebnisse häufig nicht ohne Weiteres möglich ist. Die Ergebnisse fallen uneinheitlich, zum Teil auch widersprüchlich und uneindeutig aus und zeigen unabhängig voneinander, dass verallgemeinernde Aussagen zu brückenbauenden (bridging) oder abgrenzenden Wirkungen (bonding) zu formulieren nicht legitim ist. Bridging- und Bonding-Ausprägungen realisieren sich fallspezifisch in einer Gemengelage verschiedener Einflussfaktoren und Kontextbedingungen und werden u. a. bestimmt von Zielsetzungen, Selbstverständnis und Strukturen der MSO, von ihrer jeweiligen ethnischen Zusammensetzung, von ihrem Verhältnis zum Herkunfts- und

\footnotetext{
${ }^{37}$ Inhalte und Qualität von Beziehungen sowie der Austausch von Ressourcen haben Implikationen für das Vorhandensein spezifischer Kapitalsorten (Nagel 2015b: 24 f.; vgl. Esser 2000: 213 ff.): Kontaktpflege als soziales Kapital; Geld- und Gütertransfer als ökonomisches Kapital; Wissenstransfer als kulturelles Kapital; soziale Dienste als Humankapital; öffentliche Fürsprache als symbolisches Kapital; Interessenvertretung als institutionelles bzw. politisches Kapital; Seelsorge als soziales und religiöses kulturelles Kapital; religiöse Heilung als religiöses kulturelles Kapital (Nagel 2015b: 24 f.)
} 
Aufnahmeland, der Dauer ihres Bestehens, vorhandenen Opportunitätsstrukturen, Wechselwirkungen mit Akteuren in ihren Umwelten sowie deren Reaktionen (Nagel 2013a, 2015a; Geißel et al. 2004: 12; Rauer 2004: 225 f.; Diehl 2004: 248; Berger et al. 2004).

Vor diesem Hintergrund resümiert Ludger Pries (2010a: 25; Herv. i. Orig.):

\begin{abstract}
„Zusammengefasst zeigt sich, dass die vorhandenen Studien zu Migrantenorganisationen in Deutschland nahelegen, den Grundsatzstreit über deren Funktion als Integrationsbrücke oder als Integrationsfalle, als identitäts- oder als integrationsfördernd durch differenziertere Perspektiven zu ersetzen. Migrantenorganisationen erfüllen in der Regel beide Aufgaben gleichzeitig [...] In welchem Mischungsverhältnis die verschiedenen Funktionen auftreten und welche Wirkungen sie entfalten, hängt nicht nur von den Organisationen selbst ab, sondern in starkem Ausmaß auch von der gesellschaftlichen Umwelt, in der sie agieren.“
\end{abstract}

Open Access Dieses Kapitel wird unter der Creative Commons Namensnennung 4.0 International Lizenz (http://creativecommons.org/licenses/by/4.0/deed.de) veröffentlicht, welche die Nutzung, Vervielfältigung, Bearbeitung, Verbreitung und Wiedergabe in jeglichem Medium und Format erlaubt, sofern Sie den/die ursprünglichen Autor(en) und die Quelle ordnungsgemäß nennen, einen Link zur Creative Commons Lizenz beifügen und angeben, ob Änderungen vorgenommen wurden.

Die in diesem Kapitel enthaltenen Bilder und sonstiges Drittmaterial unterliegen ebenfalls der genannten Creative Commons Lizenz, sofern sich aus der Abbildungslegende nichts anderes ergibt. Sofern das betreffende Material nicht unter der genannten Creative Commons Lizenz steht und die betreffende Handlung nicht nach gesetzlichen Vorschriften erlaubt ist, ist für die oben aufgeführten Weiterverwendungen des Materials die Einwilligung des jeweiligen Rechteinhabers einzuholen.

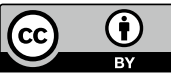

